\title{
Simulation Analysis of Cardiac Muscle Isotonic Contractions at Different Pre- and Afterloads
}

\author{
Natalie S. Schneider ${ }^{* 1,2}$ and Akira Amano ${ }^{2}$ \\ ${ }^{I}$ Cell/Biodynamics Simulation Project Kyoto University, Japan; ${ }^{2}$ Department of Systems Science, Graduate School of \\ Informatics, Kyoto University, Kyoto, Japan
}

\begin{abstract}
Since regulation of cardiac muscle contraction is complex, many simulation studies have been conducted to systematically analyze regulatory mechanisms underlying the force-velocity relationship. However, past studies were performed with models lacking detailed thin filament activation despite its essential regulatory role. Here a novel cardiac muscle contraction model is presented that considers troponin $\mathrm{C}$, troponin I and tropomyosin for thin filament activation coupled with the cross-bridge $(\mathrm{Xb})$ cycle, and in addition, includes a potential Frank-Starling mechanism and simple $\mathrm{Xb}$ mechanics. This model was employed to elucidate load and sarcomere length-dependence of the thin filament and Xb kinetics during muscle shortening and relaxation. Simulation analysis of afterloaded isotonic contractions performed at various preloads revealed that at medium to high load the peak $\mathrm{Xb}$ concentration, regulated by a load-dependent change of the ADP release rate, is the major factor in determining the end-systolic half sarcomere length, whereas the velocitydependent $\mathrm{Xb}$ force only shows a small influence. At low load, shortening velocity is regulated through an increase in the rate of the tropomyosin conformational change as for all preloads the same $\mathrm{Xb}$ concentration is attained. Shorteninginduced cooperative deactivation was caused by the included Frank-Starling mechanism. An analysis of newly suggested relaxation mechanisms showed the significance for an increased thin filament deactivation with troponin I pulling tropomyosin to the "off" position having a greater impact than titin restoring force assumed to disrupt the tropomyosin structure. A combination of this model with the myocyte Kyoto Model satisfactorily reproduced isotonic contraction time courses from guinea pig myocytes.
\end{abstract}

Keywords: Afterloaded isotonic contraction, muscle contraction modeling, force-velocity relationship, relaxation, titin, shortening-induced deactivation.

\section{INTRODUCTION}

Preload (PL) and afterload (AL), which are constantly changing in the intact heart, are important controllers of cardiac performance. In the heart the PL is indicated by the enddiastolic volume or pressure [1]. On the myocyte level the given PL establishes the maximum possible resting sarcomere length (SL), the SL from where contraction starts. This end-diastolic SL is the key in determining the active tension. As expressed in the length-tension relationship (LTR) the greater the initial SL, the higher the tension, which is the basis of the Frank-Starling law of the heart. The AL is the load sensed by the muscle after it starts to contract. For an efficient pump activity the heart has to adjust continually to the changing load. How load-dependent shortening and relaxation is regulated is still not completely understood. The force-velocity relationship (FVR) obtained from isotonic contractions, i.e., muscle shortening against a constant load, has been extensively studied for more than half a century by experiments [2-6] and simulations [6-10]. For a given PL with increasing AL velocity decreases in a hyperbolic manner. This is mainly the result of the interaction

*Address correspondence to this author at Department of Systems Science, Graduate School of Informatics, Kyoto University, Kyoto 606-8501, Japan; Tel.: 81-75-753-3375; Fax: 81-75-753-3375;

E-mail: nati@biosim.med.kyoto-u.ac.jp between cross-bridge $(\mathrm{Xb})$ mechanics and $\mathrm{Xb}$ kinetics known as mechano-chemical coupling.

Simulations with contraction models are a desirable method to determine potential rate limiting steps and regulatory mechanisms in the interaction between actin and myosin during shortening and relaxation due to an easy and systematic way to test rate parameter values. While many simulation studies which analyze the FVR have been published, the majority of the utilized models consider only the $\mathrm{Xb}$ cycle and neglect the thin filament and its activation state [8-10]. However, some models still include $\mathrm{Ca}^{2+}$ binding to troponin $\mathrm{C}(\mathrm{TnC})$ [11-13]. Since thin filament activation is an important part of muscle contraction, its influence cannot be ignored. In this study a cardiac muscle contraction model [14], which consists of separate steps for $\mathrm{Ca}^{2+}$ binding to $\mathrm{TnC}$ and the conformational changes of troponin I (TnI) and tropomyosin $(\mathrm{Tm})$, in addition to the $\mathrm{Xb}$ cycle, was combined with simple $\mathrm{Xb}$ mechanics [11]. The model further includes a Frank-Starling mechanism and various mechanisms to express cooperativity in the activation of the thin filaments [14]. A major aim of this study was to use this, compared to other models, rather biological model for an analysis of regulatory mechanisms to advance the understanding in a field difficult to explore by experiments. Simulations of afterloaded isotonic contractions were performed in a quantitative way to analyze how different PLs and ALs affect thin fila- 
ment and $\mathrm{Xb}$ kinetics rate parameters and to determine the rate limiting step and its changes with load during shortening and relaxation. Furthermore, the effect of the Frank-Starling mechanism in isotonic contractions was analyzed. Potential relaxation mechanisms were tested.

Simulation results revealed that at medium to high loads the ADP release rate was the rate limiting step and determined the peak $\mathrm{Xb}$ concentration ([Xb]) which established the end-systolic SL, whereas at low load the Tm conformational change rate was rate limiting. Shortening-induced cooperative deactivation, for which so far a full mechanism is unaccounted for [15], could be explained by the inserted Frank-Starling mechanism. Moreover, relaxation velocity was more affected by the thin filament deactivation speed than a faster $\mathrm{Xb}$ detachment. Finally, the contraction model was designed to be easily combined with a myocyte model, as validated here using the Kyoto Model [16]. Its biological complexity allows for a precise change of rate parameters making the model suitable for future simulation studies of various pathophysiological conditions.

\section{METHODS}

\section{Model Structure and Characteristics}

In this study, a cardiac muscle contraction model previously used for an isometric contraction analysis [14], was extended for an analysis of isotonic contractions. This model shown in Fig. (1A) includes $\mathrm{Ca}^{2+}$ induced activation of the thin filaments and the $\mathrm{Xb}$ cycle and consists of seven states of a regulatory unit (RU), i.e., seven actin molecules, one $\mathrm{Tm}$ and one troponin (Tn) complex composed of $\mathrm{TnC}, \mathrm{TnI}$ and TnT. RUA_MADPPi and RUA_MADP represent strong, i.e., force-generating, $\mathrm{Xb}$ states with both showing the same force. Instead, RUAMADPPi is a weak $\mathrm{Xb}$ state, which produces no force. Throughout the text a strong $\mathrm{Xb}$ is referred to as $\mathrm{Xb}$ and the sum of the two strong $\mathrm{Xb}$ states as $[\mathrm{Xb}]$. Included in the model is a potential Frank-Starling mechanism as follows [14]: A SL-dependent change of the interfilament lattice spacing due to titin-based radial force modulates the $[\mathrm{Xb}]$ which affects the conformational change of TnI. A rise in the $[\mathrm{Xb}]$ results in a strong reduction of $\mathrm{k}_{\text {offl }}$, the rate for the conformational change of TnI to the "off" state, and therefore prevents the back reaction. This mechanism provides a link between the strong $[\mathrm{Xb}]$ and the $\mathrm{Ca}^{2+}$ sensitivity of the thin filaments. Furthermore, it was assumed that only one myosin head can be bound per RU [14] as justified by calculations [17] stating that under fully activated isometric conditions only $0.37-0.75$ myosin heads are bound per RU in the case that only one of both myosin heads of the molecule can be bound at the same time.

\section{Mathematical Model}

The complete set of equations for the model is given below. New mechanisms are described in detail while those previously explained [14] are briefly depicted. All parameters are defined in the glossary (APPENDIX). Constant parameter values are shown in Table $\mathbf{1}$.

\section{Passive Tension}

For a given PL the end-diastolic SL is determined by the passive tension $\left(\mathrm{F}_{\mathrm{p}}\right)$. In the model the slack length $(\mathrm{PL}=0)$

A

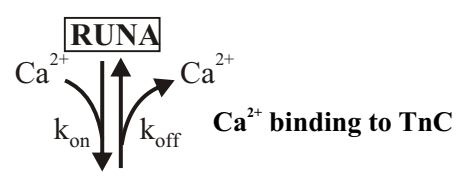

RUTCaoff

$\mathrm{k}_{\text {onI }}||_{\mathrm{k}_{\text {off }}}^{\text {conformational change }}$ of TnI

RUTCaon conformational change

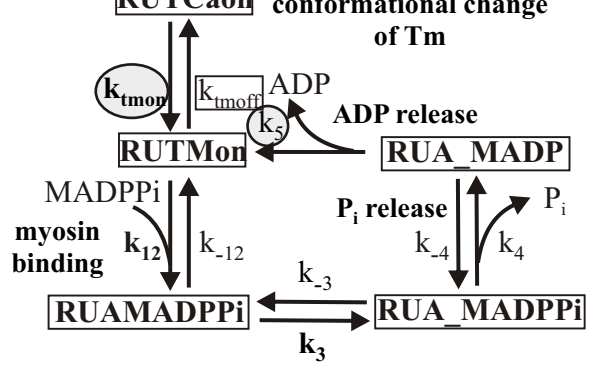

force generation
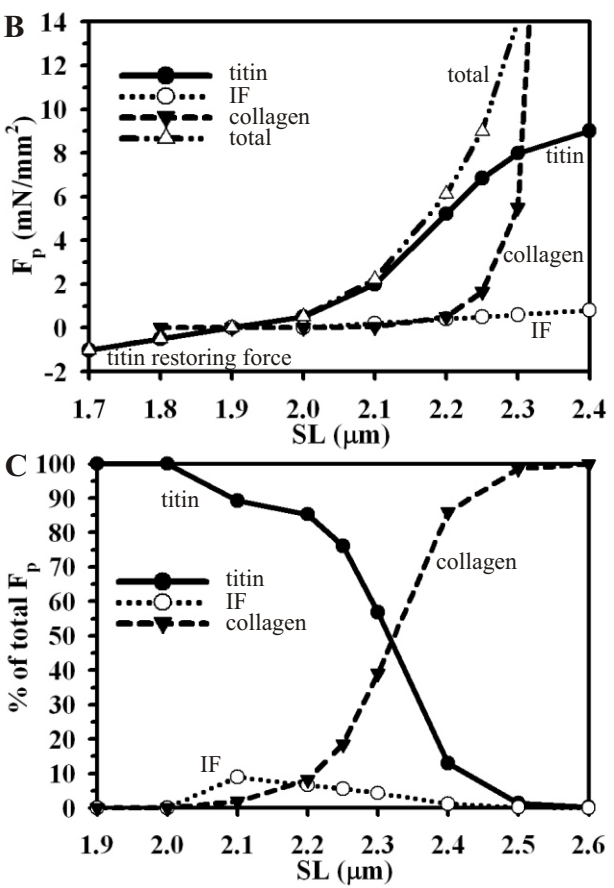

Fig. (1). Cardiac muscle contraction model. The state diagram for the model is shown in panel $\mathbf{A}$. The model is composed of seven different states of a RU (RUNA: not activated RU; RUTCaoff: $\mathrm{Ca}^{2+}$ bound to $\mathrm{TnC}$ with $\mathrm{TnI}$ still bound to A; RUTCaon: TnI released from A; RUTMon: Tm in the activated conformation; RUAMADPPi: $\mathrm{M}$ with a hydrolyzed ATP bound to A of a activated $\mathrm{RU}=$ weak $\mathrm{Xb}$; $\mathrm{M}$ pocket for $\mathrm{P}_{\mathrm{i}}$ closed; RUA_MADPPi: strong $\mathrm{Xb}, \mathrm{M}$ pocket for $\mathrm{P}_{\mathrm{i}}$ open; RUA_MADP: strong $\mathrm{Xb}, \mathrm{P}_{\mathrm{i}}$ released from the acto-myosin complex). The bold rate parameters $\mathrm{k}_{\text {off }}, \mathrm{k}_{\text {tmon }}$ and $\mathrm{k}_{3}$ are changing due to cooperativity. $k_{12}$ depends on titin-based radial force. The rate parameters indicated by a circle $\left(\mathrm{k}_{\mathrm{tmon}}, \mathrm{k}_{5}\right)$ are load-dependent. The boxed rate parameters $\left(\mathrm{k}_{\text {tmoff }}, \mathrm{k}_{\text {offl }}\right)$ are subject to changes during relaxation. Panel B depicts the SL-dependent changes of $F_{p}$. Panel $\mathbf{C}$ shows how much each contributor effects $\mathrm{F}_{\mathrm{p}}$ at a given SL above slack length. 
was set to $1.9 \mu \mathrm{m}$ in accordance to experimental data from guinea pig cardiomyocytes [18]. $\mathrm{F}_{\mathrm{p}}$ was split into three parts, one for each molecular contributor, namely titin and the intermediate filaments, which are intracellular contributors, and collagen with an extracellular action (Fig. 1B). The degree of contribution to $F_{p}$ was fit to experimental data from rat trabeculae [19] (Fig. 1C). Titin accounts for over $70 \%$ of $\mathrm{F}_{\mathrm{p}}$ from slack length to $2.25 \mu \mathrm{m}$. This percentage drops to below $20 \%$ at $2.4 \mu \mathrm{m}$. Intermediate filaments contribute $10 \%$ from slack length to $2.1 \mu \mathrm{m}$, which decreases to $3 \%$ at 2.3 $\mu \mathrm{m}$. Collagen prevents the sarcomere from overstretching while playing a minor role at the physiological SL range. Collagen contribution rises slowly from $0 \%$ at $2 \mu \mathrm{m}$ to about
$20 \%$ at $2.25 \mu \mathrm{m}$, but reaches over $80 \%$ at about $2.4 \mu \mathrm{m}$. Titin-based restoring force is responsible for $F_{p}$ below slack length [20,21], but so far could not be quantitatively measured. A linear equation was chosen to describe the half SL-F relation for intermediate filaments $\left(\mathrm{F}_{\mathrm{pIF}}\right)$.

For $\mathrm{L}>0.001 \mathrm{~mm}$ :

$$
F_{p I F}=4.0\left(L / L_{0}\right)-4.0
$$

For $\mathrm{L} \leq 0.001 \mathrm{~mm}$ :

$$
F_{p I F}=0
$$

with $\mathrm{L}$ the half $\mathrm{SL}$ and $\mathrm{L}_{0}$ a normalization factor. All $\mathrm{F}_{\mathrm{p}}$ fractions are given in $\mathrm{mN} / \mathrm{mm}^{2}$.

The data for collagen-based $F_{p}\left(F_{p E x}\right)$ were fitted to an exponential function:

Table 1. Numerical Values of Parameters

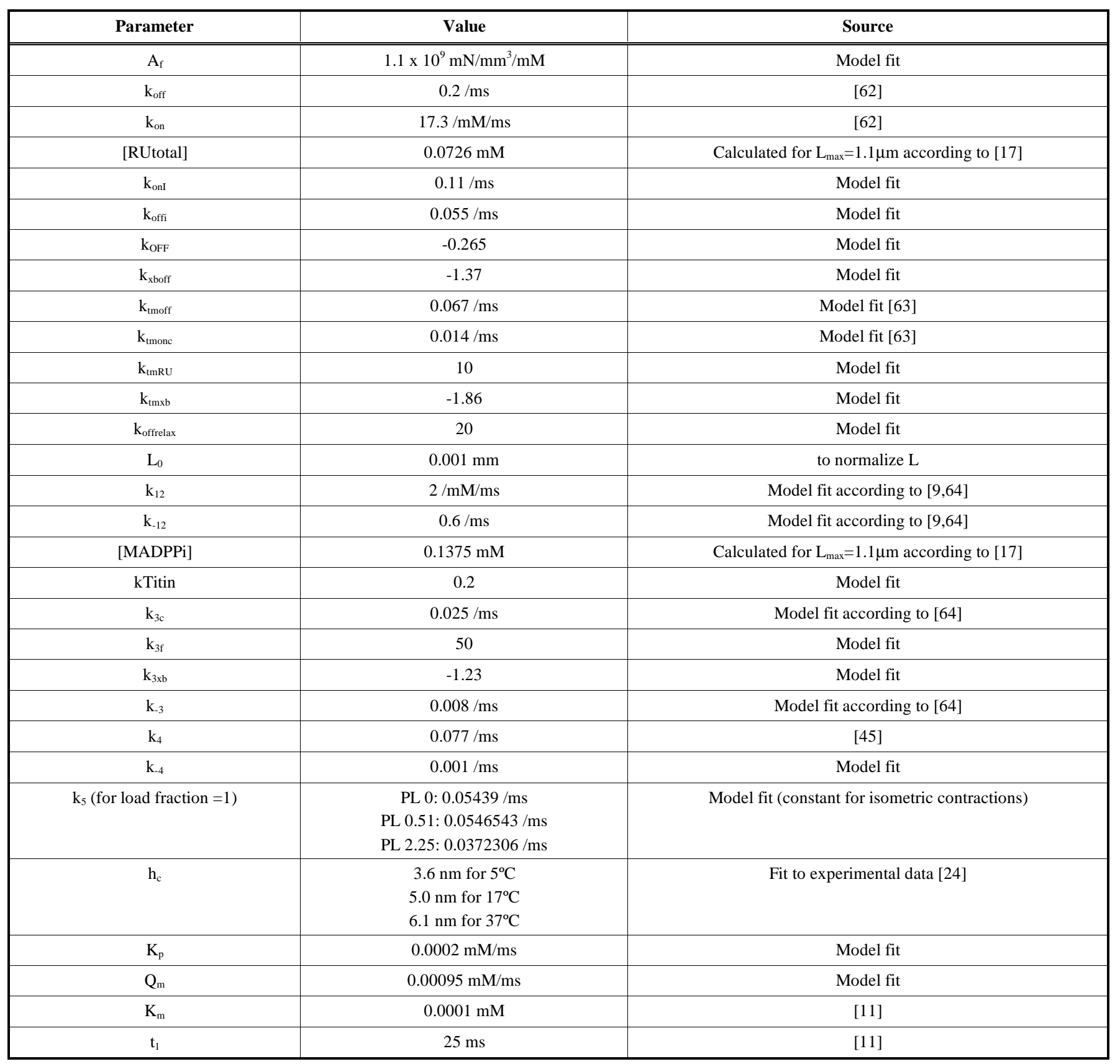


For $\mathrm{L} \geq 0.001 \mathrm{~mm}: F_{p E x}=0.20 / 47.84 \times\left(e^{47.84 \cdot\left(L / L_{0}-1\right)}-1\right)$

For $\mathrm{L}<0.001 \mathrm{~mm}: F_{p E x}=0$

Titin-based $\mathrm{F}_{\mathrm{p}}\left(\mathrm{F}_{\mathrm{p} T \mathrm{itin}}\right)$ was separated into passive tension measured above slack length and titin's restoring force below slack length:

For $\mathrm{L}<0.001 \mathrm{~mm}$ :

$F_{\text {pTitin }}=\frac{9.3323}{0.05 / 0.95 \times\left(1+e^{(1.0924-1) / 0.0324}\right)} \cdot\left(L /\left(L_{0} \times 0.95\right)-1\right)$

For $\mathrm{L} \geq 0.001 \mathrm{~mm}$ :

$$
F_{p T i t i n}=\frac{9.3323}{1+e^{\left(\left(-L / L_{0}\right)+1.0924\right) / 0.0324}}
$$

The total $\mathrm{F}_{\mathrm{p}}$ is given by: $\quad F_{p}=F_{p I F}+F_{p T i t i n}+F_{p E x}$

\section{Cross-bridge Kinetics}

The sum of all RUs (RUTotal) is given by:

$[$ RUtotal $]=[$ RUNA $]+[$ RUTCaoff $]+[$ RUTCaon $]+[$ RUTMon $]+$ $[R U A M A D P P i]+\left[R U A \_M A D P P i\right]+\left[R U A_{-} M A D P\right]$

The net rate of $\mathrm{Ca}^{2+}$ binding to $\mathrm{TnC}\left(\mathrm{Q}_{\mathrm{CaB}}\right)$ is calculated as follows:

$Q_{\text {CaB }}=k_{\text {on }}[R U N A]\left[\mathrm{Ca}^{2+}\right]-k_{\text {off }}[$ RUTCaoff $]$

The net rate for the conformational change of $\mathrm{TnI}\left(\mathrm{Q}_{\mathrm{TCaA}}\right)$ is expressed as:

$Q_{\text {TCaA }}=k_{\text {onI }}[$ RUTCaoff $]-k_{\text {offI }} \times k_{\text {relaxTnI }}[$ RUTCaon $]$

The rate parameter for the change of TnI to the "off" conformation $\left(\mathrm{k}_{\text {offt }}\right)$ changes due to positive cooperativity caused by neighboring activated RUs (RUA) (first term) and strong $\mathrm{Xbs}$ (second term):

$k_{\text {off }}=k_{\text {off }}\left[1+k_{\text {OFF }}([R U A] /[R U \text { total }])\right]^{2} \times$

$\left[1+k_{x b o f f}\left(\left[R U A \_M A D P P i\right]+\left[R U A \_M A D P\right]\right) /[R U \text { total }]\right]^{4.4}$

with

$[R U A]=[$ RUTCaon $]+[$ RUTMon $]+[R U A M A D P P i]+$

$\left[R U A_{-} M A D P P i\right]+\left[R U A_{-} M A D P\right]$

The factor $k_{\text {relaxTnI }}$ enhances relaxation at low load (see section "Relaxation Mechanisms").

The net rate for the conformational change of $\mathrm{Tm}\left(\mathrm{Q}_{\mathrm{TMA}}\right)$ is given by:

$Q_{T M A}=k_{\text {tmon }} \times k_{\text {loadTM }}[$ RUTCaon $]-k_{\text {tmoff }} \times$ titinRF $\times k_{\text {offTnI }}$

[RUTMon]

The Tm conformational change rate to the "on" state given by $\mathrm{k}_{\mathrm{tmon}}$ is subject to positive cooperativity due to activated RUs (first and second term in Eq. 11) and negative cooperativity due to strain caused by strong Xbs (third term):

$$
\begin{aligned}
& k_{\text {tmon }}=k_{\text {tmonc }}\left(\frac{[\text { RUTCaon }]}{[\text { RUtotal }]}\right) \times\left[1+k_{\text {tmRU }}\left(\frac{[\text { RUTMon }]}{[\text { RUtotal }]}\right)\right]^{2} \times \\
& {\left[1+k_{\text {tmx }} \frac{([\text { RUA_MADPPi }]+[\text { RUA_MADP }])}{[\text { RUtotal }]}\right]^{2}}
\end{aligned}
$$

The factor $\mathrm{k}_{\text {loadTM }}$ enhances the Tm conformational change at low load shortening (see section "Simulations of Afterloaded Isotonic Contractions"). The factors titinRF and $\mathrm{k}_{\mathrm{offTn}}$ speed up relaxation (see section "Relaxation Mechanisms").

The net rate of the myosin binding step $\left(\mathrm{Q}_{\mathrm{MB}}\right)$ resulting in the formation of weak Xbs is calculated as follows:

$Q_{M B}=k_{12} \times$ KTitin $[M A D P P i] \times$

$[R U T M o n]_{\text {eff }}-k_{-12}[R U A M A D P P i]$

The fraction of activated RUs overlapping with the myosin filaments called effective fraction is given by:

$[\text { RUTMon }]_{\text {eff }}=\alpha[$ RUTMon $]$

The sarcomere overlap function $\alpha$ describes the SLdependent change of the myosin filament fraction which overlaps with the actin filament:

For $\mathrm{L}<1 \mu \mathrm{m}$ :

$$
\alpha=1.5\left(L / L_{0}\right)-0.5
$$

For $\mathrm{L} \geq 1 \mu \mathrm{m}$ and $\mathrm{L} \leq 1.1 \mu \mathrm{m}$ :

$\alpha=1.0$

For $\mathrm{L}>1.1 \mu \mathrm{m}$ :

$$
\alpha=-1.6\left(L / L_{0}\right)+2.76
$$

The titin function (KTitin; Eq. 15) expresses the SLdependent change of the spacing between the actin and myosin filaments caused by titin-based radial force in the physiological SL range of the heart (Eq. 16) and the damage of titin at SLs greater than $2.2 \mu \mathrm{m}$ (Eq. 17) [14]. The latter part (Eq. 17) is not relevant concerning the physiological working range of the heart. In contrast to nature, in the model titin damage is reversible.

$$
\begin{aligned}
& \text { KTitin }=\left\{\begin{array}{cc}
f_{\text {titinNormal }} & (L \leq 1.1 \mu m) \\
f_{\text {titinDamaged }} & (L>1.1 \mu \mathrm{m})
\end{array}\right. \\
& f_{\text {titinNormal }}=k \text { Titin } \frac{6.8753}{1+e^{\left(\left(-L / L_{0}\right)+0.9601\right) / 0.0449}} \\
& f_{\text {titinDamaged }}=k \text { Titin }\left(-31.0\left(L / L_{0}\right)+40.0\right)
\end{aligned}
$$

The force generating step $\left(\mathrm{Q}_{\mathrm{Fgen}}\right)$ is characterized by a fast conformational change of the actin bound myosin, which opens the $\mathrm{P}_{\mathrm{i}}$ binding pocket and results in a strong $\mathrm{Xb}$ state. The net rate is given by:

$Q_{\text {Fgen }}=k_{3}[R U A M A D P P i]-k_{-3}\left[R U A M_{-} A D P P i\right]$

The rate parameter for this conformational change $\left(\mathrm{k}_{3}\right)$ changes due to positive cooperativity caused by activated RUs (first term in Eq. 19) and negative cooperativity caused by strong Xbs (second term) as follows:

$$
\begin{aligned}
& k_{3}=k_{3 c}\left[1+k_{3 f}\left(\frac{[\text { RUTCaon }]_{\text {eff }}+[\text { RUTMon }]_{\text {eff }}+[\text { RUAMADPPi }]}{[\text { RUtotal }]}\right)\right]^{2} \times \\
& {\left[1+k_{3 x b} \frac{\left([\text { RUA_MADPPi }]+\left[R U A_{-} \text {MADP }\right]\right)}{[\text { RUtotal }]}\right]^{2}} \\
& {[\text { RUTCaon }]_{\text {eff }}=\alpha[\text { RUTCaon }]}
\end{aligned}
$$

The net rate of the $\mathrm{P}_{\mathrm{i}}$ release $\left(\mathrm{Q}_{\mathrm{PiR}}\right)$ is expressed as: 
$Q_{P i R}=k_{4}\left[R U A_{-} M A D P P i\right]-k_{-4}\left[R U A_{-} M A D P\right]$

The net rate of the ADP release $\left(\mathrm{Q}_{\mathrm{ADPR}}\right)$ is given by:

$Q_{A D P R}=k_{5}\left[R U A_{-} M A D P\right]$

In isometric contractions (load fraction 1) the ADP release is the rate limiting step of the $\mathrm{Xb}$ cycle with a constant $\mathrm{k}_{5}$ value depending on the PL (Table 1). In isotonic contractions the $\mathrm{k}_{5}$ value is PL and AL-dependent (see section "Simulations of Afterloaded Isotonic Contractions").

The rate changes for the different RU species and $\left[\mathrm{Ca}^{2+}\right]$ are determined as follows:

$$
\begin{aligned}
& d[\text { RUTCaoff }] / d t=Q_{C a B}-Q_{T C a A} \\
& d[\text { RUTCaon }] / d t=Q_{T C a A}-Q_{T M A} \\
& d[\text { RUTMon }] / d t=Q_{T M A}-Q_{M B}+Q_{A D P R} \\
& d[\text { RUAMADPPi }] / d t=Q_{M B}-Q_{F g e n} \\
& d\left[R U A_{-} M A D P P i\right] / d t=Q_{F g e n}-Q_{P i R} \\
& d\left[R U A_{-} M A D P\right] d t=Q_{P i R}-Q_{A D P R} \\
& d\left[\mathrm{Ca}^{2+}\right] / d t=-Q_{C a B}
\end{aligned}
$$

The active force $\left(\mathrm{F}_{\mathrm{b}}\right)$ is proportional to the number of developed $\mathrm{Xbs}$ with $\mathrm{A}_{\mathrm{f}}$ being the force factor [11].

$F_{b}=A_{f}\left(\left[R U A_{-} M A D P P i\right]+\left[R U A_{-} M A D P\right]\right) \times(L-X)$

The $(\mathrm{L}-\mathrm{X})$ term implies a change of the average $\mathrm{Xb}$ force with load (see Eq. 32 below). The external force $\left(\mathrm{F}_{\mathrm{ext}}=\right.$ load $)$ is defined as

$F_{e x t}=F_{b}+F_{p}$

\section{Cross-bridge Mechanics and Active Force}

For the $\mathrm{Xb}$ mechanics the equation developed by Negroni and Lascano [11] was used, which describes the velocity for the movement of the mobile $\mathrm{Xb}$ end $(\mathrm{dX} / \mathrm{dt})$ :

$$
d X / d t=B \times\left(h-h_{c}\right) \quad \text { with } \quad h=L-X
$$

where $\mathrm{h}$ is defined as the $\mathrm{Xb}$ elongation, $\mathrm{h}_{\mathrm{c}}$ the steady state $\mathrm{Xb}$ elongation, $\mathrm{X}$ the part of $\mathrm{L}$, which is not covered by $\mathrm{h}$, and $\mathrm{B}$ as a proportionality factor. Analysis of force step simulations revealed a change of the $h_{c}$ value with temperature and a B value change with load as shown in the next section.

\section{Force Step Simulations to Adjust the $h_{c}$ and B Parameter Values}

A force step, which is initiated through a fast reduction of load from a muscle in the tetanic steady state, results in SL shortening characterized by four different phases [5]. Xb mechanics is clearly distinct from the kinetics in the elastic phase (P1) and the fast shortening phase (P2). X-ray diffraction data from frog skeletal muscle demonstrated that during P1 and P2 myosin heads move towards the center of the sarcomere, but no detachment and reattachment of Xbs was found [22]. To represent this behavior, in the force step simulations during $\mathrm{P} 1$ and $\mathrm{P} 2$ the myosin binding rate parameter $\left(k_{12}\right)$ and the ADP release rate $\left(k_{5}\right)$ were set to zero. Simulations of P1 and P2 allowed allocating a physiological value and a biological meaning to the parameters $h_{c}$ and $B$ of the mechanics equation (Eq. 32). Since the only available experimental force step data from frog skeletal muscle recorded at $5^{\circ} \mathrm{C}$ [5] were not appropriate for model adjustment, the slow shortening phase (P3) and the steady state shortening phase (P4) were not analyzed.

Simulations were essentially performed as reported for force steps of frog skeletal muscle fibers [5]. First the tetanic steady state values for all variables and parameters were determined using the isometric model with a constant $\left[\mathrm{Ca}^{2+}\right]=$ $2 \mu \mathrm{M}$ and $\mathrm{L}=1.05 \mu \mathrm{m}$. A force step simulation experiment was started from the tetanic steady state through a change from the isometric contraction model, i.e., $\mathrm{F}_{\mathrm{ext}}=0$ and $F_{b}=-F_{p}$, to the isotonic model. Within one time step $(0.001$ $\mathrm{ms}) \mathrm{F}_{\text {ext }}$ was set to account for the desired load as follows:

$F_{\text {ext }}=$ loadfraction $\cdot T_{0}$

with $\mathrm{T}_{0}$ being the tetanic tension. In contrast, in the experiments the force step took $0.150 \mathrm{~ms}$ to be complete [5]. Simulation results for the $\mathrm{P} 1$ and $\mathrm{P} 2 \mathrm{~L}$ shortening time courses for $5^{\circ} \mathrm{C}$ and $37^{\circ} \mathrm{C}$ are shown in Fig. (2A) and (2B), respectively.

\section{Temperature Dependence of the Phase 1 Elastic Stroke}

$\mathrm{X}$-ray diffraction data from frog skeletal muscle fibers revealed that a rise in temperature from $0^{\circ} \mathrm{C}$ to $17^{\circ} \mathrm{C}$ causes the light-chain domain of the myosin heads to tilt axially, and the interference distance to decrease [23]. This observation led to our hypothesis that the length of the tilting myosin heads might be represented in the mechanics equation (Eq. 32) as $h_{c}$, and therefore, $h_{c}$ should increase with an increase in temperature. In force step simulations a variation of $h_{c}$ resulted solely in a change of $\mathrm{L}_{1}$, the length change due to the elasticity of the muscle. From the simulation data $L_{1}$ was taken as the length of $\mathrm{L}$ immediately after the force step as indicated in Fig. (2A). Experimental data revealed a temperature dependence for $L_{1}$ (Fig. 2C, symbols) [24]. The $h_{c}$ value, which was determined by simulations to match the experimental $\mathrm{L}_{1}$ value for a specific load and temperature, could reproduce $L_{1}$ values for other load fractions at the same temperature (Fig. 2C, lines). For a good match to experimental data, $h_{c}$ was determined as $3.6 \mathrm{~nm}$ for $5^{\circ} \mathrm{C}$ and 5.0 $\mathrm{nm}$ for $17^{\circ} \mathrm{C}$. Due to the lack of experimental data from cardiac muscle, $h_{c}$ was adjusted to match data obtained from skeletal muscle, although cardiac muscle was found to be stiffer than skeletal muscle fibers [19,25].

A change of $h_{c}$ in the isometric contraction model [14] inserted into the Kyoto Model [16] (see section "Simulations and Data Analysis") produced a change of $F_{b}$ (Fig. 2D). However, the rise of $F_{b}$ with an increase of $h_{c}$ was not accompanied by a change of the [Xb] (insert in Fig. 2D). Under isometric conditions, $h_{c}$ is equal to (L-X) (Eq. 30), and consequently, a higher $h_{c}$ results in a larger $F_{b}$. This coincides with experimental findings that show a rise in active tension with increasing temperature without a change in stiffness [23,26]. It was concluded that the higher force is due to the higher strain in the head producing a higher force per Xb [23]. 

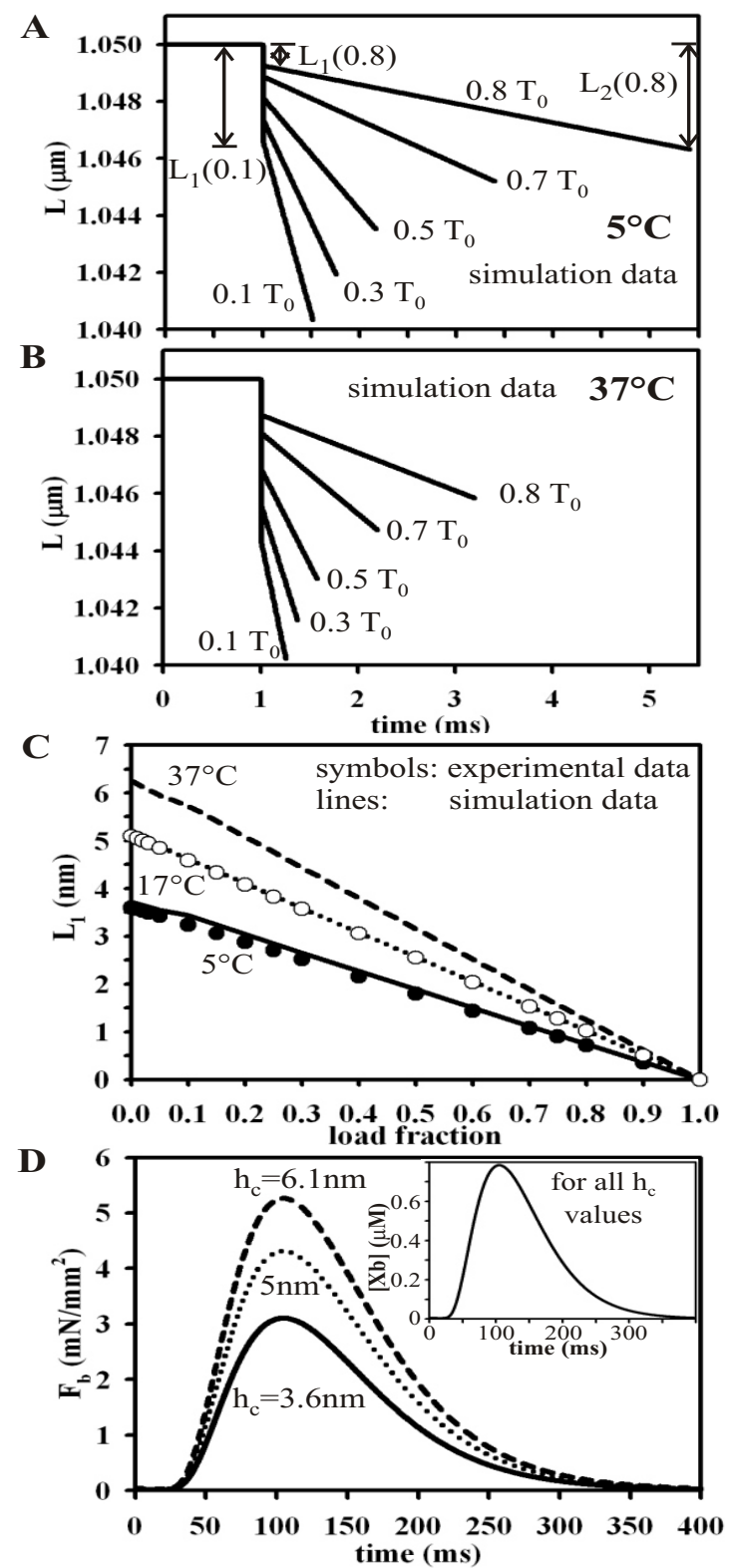

Fig. (2). Temperature dependence of the force step elastic shortening phase (P1). The $\mathrm{L}$ shortening time course from simulations of force steps (P1 and $\mathrm{P} 2$ ) is shown in panel $\mathbf{A}$ for $5^{\circ} \mathrm{C}$ and in $\mathbf{B}$ for $37^{\circ} \mathrm{C}$. The numbers indicate load fractions. In panel $\mathbf{A} \mathrm{L}_{1}$ for load fraction 0.1 and 0.8 and $\mathrm{L}_{2}$ for load fraction 0.8 are indicated by arrows. In panel $\mathbf{C}$ experimental data from frog skeletal muscle fibers (symbols) [24] for the load and temperature dependence of $\mathrm{L}_{1}$ are compared to simulation results (lines). Panel $\mathbf{D}$ shows the alteration of the $\mathrm{F}_{\mathrm{b}}$ time course from isometric twitch contractions $(L=1.05 \mu \mathrm{m})$ with a change of $h_{c}$. Data were obtained from the isometric model inserted into the Kyoto Model [16]. The insert in D shows the identical $[\mathrm{Xb}]$ time course for all $\mathrm{h}_{\mathrm{c}}$ values.

A linear increase of $h_{c}$ with temperature causes a linear rise of $\mathrm{F}_{\mathrm{b}}$ with temperature. However, experiments showed that $\mathrm{F}_{\mathrm{b}}$ rises linearly with temperature up to about $17-20^{\circ} \mathrm{C}$, but reaches a plateau at higher temperatures [27]. Therefore, $h_{c}$ for $37^{\circ} \mathrm{C}$, the desired temperature for the contraction model, was set to $6.1 \mathrm{~nm}$ to match the experimentally found non-linear $\mathrm{F}_{\mathrm{b}}$-temperature relation. In conclusion, simulation results allow attributing $h_{c}$ to the length of the myosin head and its temperature-dependent elastic changes.

\section{The Factor B Determines Phase 2}

P2 is characterized by a fast linear shortening of L (Fig. $\mathbf{2 A}$ and $\mathbf{2 B}$ ) to $\mathrm{L}_{2}$, the sum of the elastic and the working stroke $\left(\mathrm{L}_{\mathrm{T}}\right)$, which are related as follows:

$L_{T}=L_{2}-L_{1}$

From the only available force step experimental data set consisting of the shortening velocity of P2 (V2) (Fig. (3A), solid line with filled circles) and $\mathrm{L}_{\mathrm{T}}$ (Fig. 3B, filled triangles) from frog skeletal muscle $\left(5^{\circ} \mathrm{C}\right)$ [5], the duration of P2 $\left(\mathrm{t}_{2}\right)$ for each load was calculated as follows (Fig. 3A, dotted line with filled circles):

$t_{2}=L_{T} / V 2$

Using $t_{2}$ the model could be adjusted to match the V2 data through a change of $B$ with load (Eq. 32), resulting in $L_{2}$ and $\mathrm{L}_{\mathrm{T}}$ for $5^{\circ} \mathrm{C}$ as depicted in Fig. (3B) $\left(\mathrm{L}_{2}\right.$ : solid line; $\mathrm{L}_{\mathrm{T}}$ : dashed line). $\mathrm{L}_{2}$ was determined from the simulation data as the amount of shortening attained at the end of P2 (Fig. 2A). In contrast, in the experiments $L_{2}$ was measured graphically using a tangent on the P3 shortening time course [5]. This method, however, results in a $\mathrm{L}_{2}$ value which is highly dependent on the P3 shortening time course. The slight divergence from experimental data is probably due to the different method used to determine $\mathrm{L}_{2}$. V2 was determined from simulation data through a linear regression analysis of a time versus L plot over the whole period of P2, which is linear (Fig. 2A and 2B). The obtained B-load relationship for $5^{\circ} \mathrm{C}$ is shown in Fig. (3C) (filled circles).

Due to the lack of $37^{\circ} \mathrm{C}$ experimental force step data from cardiac muscle which include $\mathrm{P} 2$, some assumptions were necessary to develop a B-load relationship. $\mathrm{L}_{\mathrm{T}}$ was set to 4 $\mathrm{nm}$ independent of load, since experiments revealed that $\mathrm{L}_{\mathrm{T}}$ gets load independent at higher temperature [24]. Although having the unit $[1 / \mathrm{ms}], \mathrm{B}$ is not equal to $1 / \mathrm{t}_{2}$. As the factor for $\mathrm{dX} / \mathrm{dt}, \mathrm{B}$ is defined as

$B=\frac{d X}{d t} \times \frac{1}{\left(L-X-h_{c}\right)}$

This equation could be discretized as follows to apply simulation data:

$B=\frac{X_{P 2 \text { end }}-X_{P 2 \text { start }}}{t_{2}} \times \frac{1}{L_{P 2 \text { mid }}-X_{P 2 \text { mid }}-h_{c}}=\frac{c}{t_{2}}$

with $\mathrm{X}_{\mathrm{P} 2 \text { start }}, \mathrm{X}_{\mathrm{P} 2 \mathrm{mid}}$ and $\mathrm{X}_{\mathrm{P} 2 \text { end }}$ being $\mathrm{X}$ at the start, middle and end of $\mathrm{P} 2$, respectively and $\mathrm{L}_{\mathrm{P} 2 \mathrm{mid}}, \mathrm{L}$ at the middle of $\mathrm{P} 2$. The value of $c$ depends on $h_{c}$ (Eq. 37) and $L_{T}$. But according to $c=B \times t_{2}, \mathrm{c}$ is valid for various $\mathrm{B}-\mathrm{t}_{2}$ combinations. This allows the calculation of $\mathrm{c}$ from preliminary simulation data, which were obtained with $\mathrm{h}_{\mathrm{c}}=6.1 \mathrm{~nm}, \mathrm{~B}$ values from $5^{\circ} \mathrm{C}$, and $\mathrm{L}_{\mathrm{T}}$ adjusted to $4 \mathrm{~nm}$ through a change of $\mathrm{t}_{2}$. To calculate 


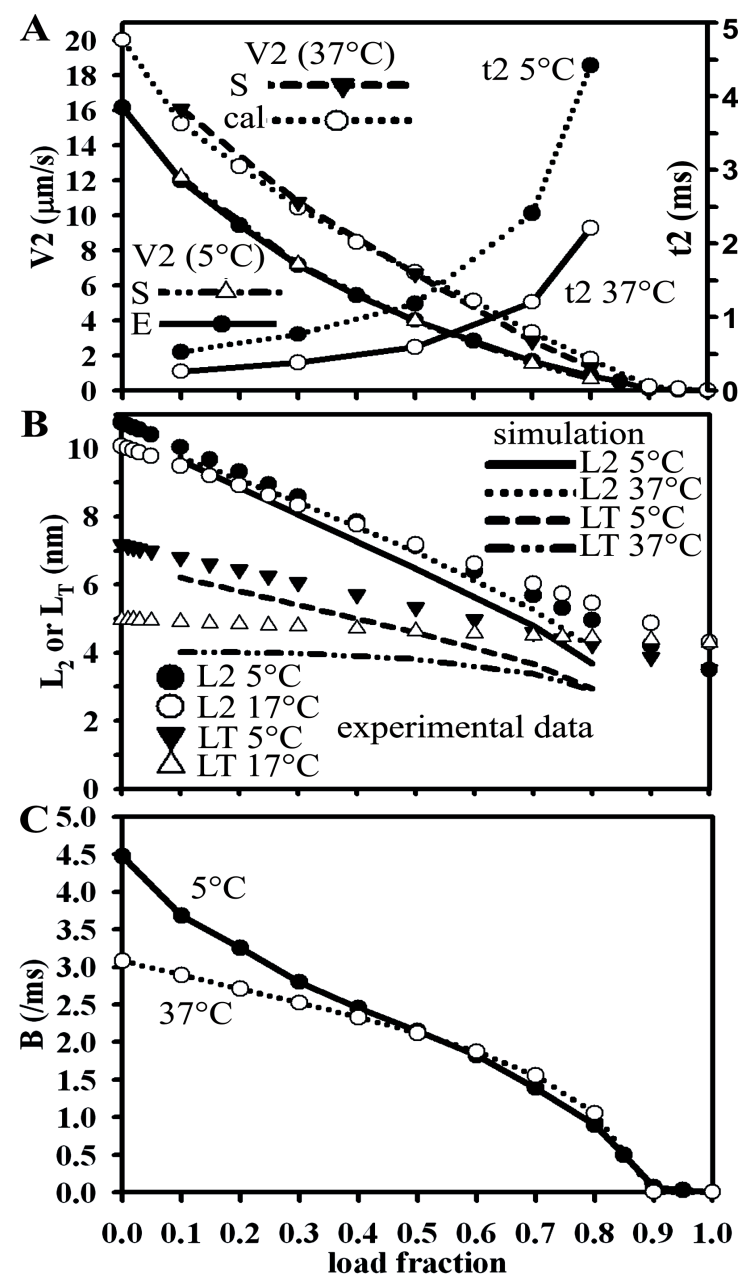

Fig. (3). Characterization of the force step fast shortening phase (P2). Panel A shows V2 data from $5^{\circ} \mathrm{C}$ frog skeletal muscle experiments [5] (solid line with filled circles, labeled E) compared to V2 data obtained from $5^{\circ} \mathrm{C}$ simulations (open triangles, labeled S) using the calculated $\mathrm{t}_{2}\left(5^{\circ} \mathrm{C}\right)$ data (dotted line with filled circles). For $37^{\circ} \mathrm{C}$ simulations the assumed $t_{2}$ data (solid line with open circles) were used. Simulation results for $\mathrm{V} 2\left(37^{\circ} \mathrm{C}\right)$ (filled triangles, labeled S) are compared to calculated V2 data (dotted line with open circles, labeled cal). Experimental data from frog skeletal muscle for $5^{\circ} \mathrm{C}$ and $17^{\circ} \mathrm{C}$ (symbols) [24] and the simulation results for $5^{\circ} \mathrm{C}$ and $37^{\circ} \mathrm{C}$ (lines) for the load dependence of $\mathrm{L}_{2}$ and $\mathrm{L}_{\mathrm{T}}$ are shown in $\mathbf{B}$. The obtained B-load relationships are depicted in $\mathbf{C}$.

the $\mathrm{B}$ values from these calculated $\mathrm{c}$ values, $\mathrm{t}_{2}$ was assumed to be $50 \%$ of $\mathrm{t}_{2}$ from frog $\left(5^{\circ} \mathrm{C}\right)$ (Fig. 3A, solid line with open circles). The dominant cardiac muscle myosin heavy chain (MHC) in healthy rodent hearts, $\alpha$, is significantly slower than the fast skeletal muscle $\mathrm{MHC}$ isoforms [28]. However, the temperature difference of $32^{\circ} \mathrm{C}$ was taken into account. From the calculated $\mathrm{c}$ and the assumed $\mathrm{t}_{2}$ values, $\mathrm{B}$ for $37^{\circ} \mathrm{C}$ was calculated $\left(\mathrm{B}=\mathrm{c} / \mathrm{t}_{2}\right)$. Then $\mathrm{V} 2$ was determined as follows (Fig. 3A, dotted line with open circles):

$$
V 2=B \cdot L_{T} / c
$$

which gave similar results to V2 determined from simulation data using $\mathrm{B}$ and $\mathrm{t}_{2}$ (Fig. 3A, dashed line with filled trian- gles). The B-load relation for $37^{\circ} \mathrm{C}$ as shown in Fig. 3C (open circles) was fitted as follows:

$$
\begin{aligned}
\text { load } \leq 0.9: & B & =\frac{3.08-3.42 \text { load }}{1-0.48 \text { load }-0.44 \text { load }^{2}} \\
\text { load }>0.9: & B & =0.006
\end{aligned}
$$

A decreasing $B$ with increasing load implies a decrease of V2 with load. A change of B with load under steady state shortening was found to increase model stability. Due to $h_{c}$, c is temperature-dependent (Eq. 37), and therefore B. Since no $\mathrm{Xb}$ attachment and detachment occurs in $\mathrm{P} 2, \mathrm{~V} 2$ is only determined by the myosin isoform. Hence, $\mathrm{B}$ represents the myosin isoform characteristic. A change of B might be necessary with an isoform shift which is sometimes found in the diseased heart [29]. $\mathrm{L}_{2}$ represents the maximum possible extension of the myosin head before detachment and is about $11 \mathrm{~nm}$ at low load independent of temperature (Fig. 3B) [24]. In conclusion, the $\mathrm{Xb}$ mechanics is satisfactorily described by Eq. 32 .

\section{Simulations of Afterloaded Isotonic Contractions}

Simulations of afterloaded isotonic contractions were performed based on cat papillary muscle experiments [2]. In these experiments the muscle was first stretched by a small weight, the PL, to a length consistent to its resting LTR. This length was then fixed, so that the muscle did not sense any additional added weight, the AL, until it started to contract. For the simulations the resting $\mathrm{L}$ was determined by setting the PL equal to $\mathrm{F}_{\text {ext }}$. A $40 \mathrm{nM}$ resting $\left[\mathrm{Ca}^{2+}\right]$ was used. The isometric model combined with a simple SR model (see section "Simulations and Data Analysis") was used to determine the maximum $F_{b}$ for the given PL. The maximum $F_{e x t}$ $\left(\mathrm{F}_{\text {extmax }}\right)$, which is the force at which the muscle cannot shorten any longer (isometric contraction), was defined as

$F_{\text {ext } \max }=F_{p}+F_{b \max }$

and used to calculate the load fractions

load $=F_{\text {ext }} / F_{\text {ext } \max }$ with $F_{\text {ext }}=P L+A L$

Shortening velocity was calculated for each time step from the change of $\mathrm{L}$ during the time step:

$$
V=\Delta L / \text { time step }
$$

The peak shortening velocity was used for the FVR. At times where L was constant, i.e., no shortening, all loaddependent parameters were set to the isometric value, i.e., load fraction $=1$. The B-load relation (Eq. 39) was used, and $\mathrm{h}_{\mathrm{c}}$ set to $6.1 \mathrm{~nm}$.

For model analysis three PLs were chosen: PL 0, i.e., 0 $\mathrm{mN} / \mathrm{mm}^{2}$ (resting $\mathrm{L}=0.95 \mu \mathrm{m} ; \mathrm{F}_{\text {extmax }}=5.91 \mathrm{mN} / \mathrm{mm}^{2}$ ); PL $0.51\left(1.0 \mu \mathrm{m} ; 8.38 \mathrm{mN} / \mathrm{mm}^{2}\right)$; PL $2.25(1.0504 \mu \mathrm{m} ; 10.89$ $\mathrm{mN} / \mathrm{mm}^{2}$ ). The availability of appropriate experimental data for model adjustment is very limited. Although cat papillary muscle experimental data for different PLs and ALs are available [2], only velocities for the muscle length changes given in $(\mathrm{mm} / \mathrm{s})$ were measured and plotted against the force in gram (Fig. 4A). Furthermore, these data were obtained 
before it became obvious that damage to the ends of papillary muscles during preparation could cause artifacts in measurements [30]. Taking the shape of the experimental FVR (Fig. 4A) as reference, data to fit the PL 2.25 model were designed (Fig. 4B, filled squares). To adjust the unloaded shortening velocity $\left(\mathrm{V}_{\max }\right)$ of the model, experimental data obtained from cat trabeculae $\left(\mathrm{V}_{\max }=8.2 \mu \mathrm{m} / \mathrm{s}, 25^{\circ} \mathrm{C}\right)[4]$ and guinea pig single myocytes $\left(\mathrm{V}_{\max }=6-12.4 \mu \mathrm{m} / \mathrm{s}, 35^{\circ} \mathrm{C}\right)$ [18] were taken as reference values. The shape of the FVR is influenced by many factors such as $\mathrm{SL},\left[\mathrm{Ca}^{2+}\right]$, temperature, the MHC isoform [29], artifacts in measurements [30] and especially, depends on the type of the experiment performed and the time velocity is measured $[2,4]$. Generally, the FVR obtained from afterloaded isotonic contractions is less hy-


Fig. (4). Force-velocity relationship. Panel A shows experimental data for the FVR obtained for various PLs and ALs from cat papillary muscle [2]. The temperature was not given, but this group performed most experiments at 25 or $30^{\circ} \mathrm{C}$. The FVR is PL dependent but plotting against the load fraction results in a single FVR (insert in A). A more hyperbolic FVR was obtained from force step experiments performed with cat trabeculae at $25^{\circ} \mathrm{C}$ (insert in $\mathbf{A}$; solid line with open circles) [4]. FVR simulation results for PL $0,0.51$ and 2.25 for the original model (M1) and the model variant which uses $\mathrm{k}_{5} \downarrow$ are shown in B. For PL 2.25, data from M1 and $\mathrm{k}_{5} \downarrow$ are identical. The insert in B shows the M1 data plotted against the load fraction. perbolic than the FVR obtained from force step experiments (insert in Fig. 4A). Due to the great variation with changing conditions, assuming physiological data for model fitting should be justified. Fitting was achieved through a variation of the ADP release rate $\left(\mathrm{k}_{5}\right)$ with different ALs. $\mathrm{k}_{5}$ was chosen since it is the rate limiting step in the $\mathrm{Xb}$ cycle, and experimental data suggest the ADP release to be loaddependent (see discussion). At load fraction 1 ( $\mathrm{AL}=8.64$ $\mathrm{mN} / \mathrm{mm}^{2}$ ) $\mathrm{k}_{5}=0.0372306 / \mathrm{ms}$ was used to give $\mathrm{F}$ which matches $\mathrm{F}_{\text {extmax }}$. At load 1 the ADP release rate should have its minimum value, since more $\mathrm{Xbs}$ are necessary to bear the load. Naturally, an increasing $\mathrm{k}_{5}$ rate parameter would be expected with a decrease in load. However, $\mathrm{k}_{5}$ rapidly decreased for load fractions smaller than 0.35 (Fig. $\mathbf{5 A}$, filled squares, labeled $\left.\mathrm{k}_{5} \downarrow\right)$. A steadily increasing $\mathrm{k}_{5}$-load curve was designed for low load as shown in Fig. (5A) (dotted line with open circles) and fitted as follows:

For load <1: $\begin{aligned} & k_{5}=0.1419-(0.2449 \text { load })+ \\ & \left(0.1892 \text { load }^{2}\right)-\left(0.0489 \text { load }^{3}\right)\end{aligned}$

For load $=1: k_{5}=0.0373206$

Using this relationship the model was then adjusted for low load to the desired shortening velocity by changing the speed of the Tm conformational change rate $\left(\mathrm{k}_{\mathrm{tmon}}\right)$, the rate limiting step in the thin filament activation, using the factor $\mathrm{k}_{\text {loadTM}}$. The $\mathrm{k}_{\text {loadTM}}$-load relationship shown in Fig. (5B) (filled triangles) was fitted as follows:

$$
\begin{aligned}
& \text { PL 2.25: For load <0.36725: } k_{\text {loadTM }}=0.9652+ \\
& 3.2044 e^{(-12.3147 \text { load })} \\
& \text { For load } \geq 0.36725: \quad k_{\text {loadTM }}=1.0
\end{aligned}
$$

The attained end-systolic $\mathrm{L}\left(\mathrm{L}_{\min }\right)$ decreased linear with a decrease in AL as depicted in Fig. (6A) (filled triangles). Experimental data from cat papillary muscle revealed that for different PLs, but for the same final loads $\left(\mathrm{F}_{\mathrm{ext}}=\mathrm{PL}+\mathrm{AL}\right)$, the muscle shortened to the same end-systolic muscle length [31]. In addition, isolated intact cardiac myocytes from guinea pig shortened to the same end-systolic cell length or $\mathrm{SL}$ at $37^{\circ} \mathrm{C}$ despite different PLs if loaded with the same total load [32]. Based on these findings, using the $\mathrm{F}_{\text {ext }}-\mathrm{L}_{\text {min }}$ relationship obtained from PL 2.25 (Fig. 6A), the models for PL 0 and PL 0.51 were fitted to match this relationship. For PL $0.51 \mathrm{k}_{5}=0.0546543 / \mathrm{ms}$ and for PL $0 \mathrm{k}_{5}=0.05439 / \mathrm{ms}$ were determined for load fraction 1. Again $\mathrm{k}_{5}$ was found to decrease at low load (Fig. 5A, filled (PL 0) and open (PL 0.51) triangles; labeled $\left.\mathrm{k}_{5} \downarrow\right)$. A constantly rising $\mathrm{k}_{5}$ with a decrease of load was designed which was appropriate for PL 0 and 0.51 (Fig. 5A, filled circles).

For load $\leq 0.8275: \quad k_{5}=0.0317+0.2035 e^{(-2.5623 \text { load })}$

For $0.8275<\operatorname{load}<1: k_{5}=0.0668-(0.0168$ load $)+$

$$
\left(0.0047 \text { load }^{2}\right)
$$

For low load both PL models were adjusted to the $\mathrm{L}_{\text {min }}$ as determined for PL 2.25 with a change of $\mathrm{k}_{\text {tmon }}$ using the factor $\mathrm{k}_{\text {loadTM }}$ (Eq. 10). The $\mathrm{k}_{\text {loadTM}}$-load relationships for PL 0 


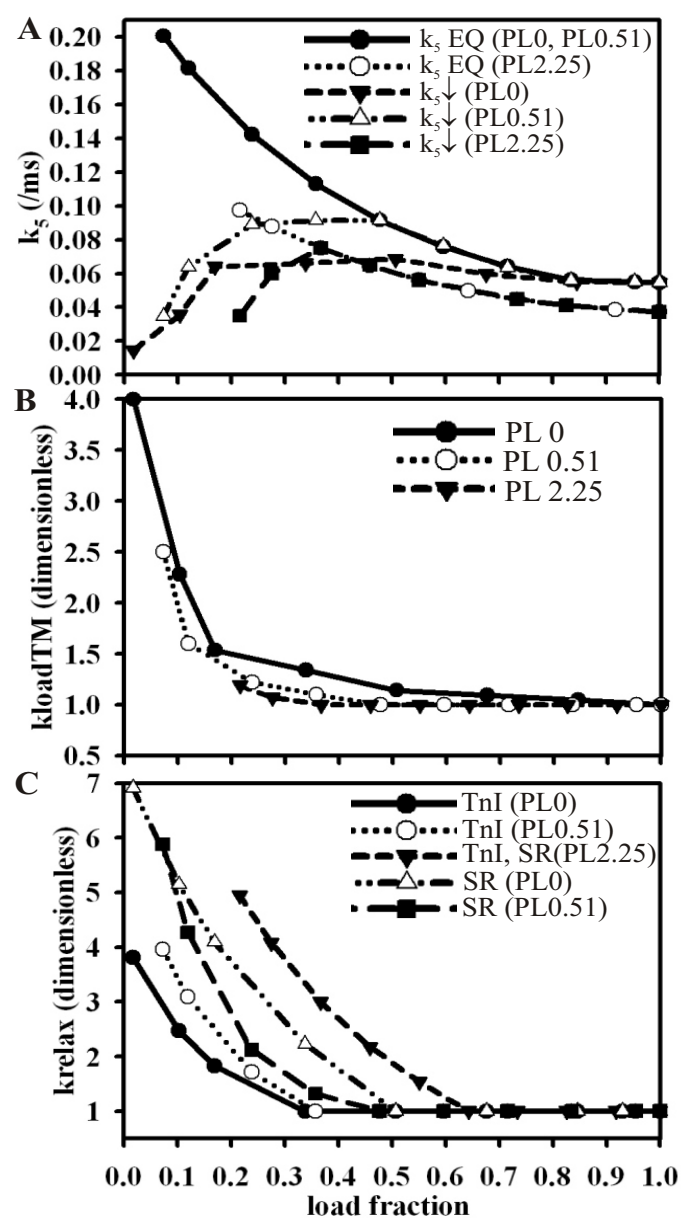

Fig. (5). Load dependence of rate parameters. The load dependence of the ADP release rate, $\mathrm{k}_{5}$, is shown in A. EQ indicates that the results are from the fitted equation, Eq. 43 (PL 2.25) and Eq. 45 (PL 0 and 0.51). Results from the fitted equations Eq. 44, Eq. 46 and Eq. 47 for the load-dependent change of the Tm conformational change rate by the factor $\mathrm{k}_{\text {loadTM }}$ are shown in $\mathbf{B}$. The loaddependent changes of the TnI conformational change to the "off" conformation $\left(\mathrm{k}_{\text {offI }}\right)$ (labeled TnI; Eq. 50-52) and the increase in the SR $\mathrm{Ca}^{2+}$ uptake (labeled SR; Eq. 57-59) during relaxation are depicted in panel $\mathbf{C}$.

(filled circles; Eq. 46) and PL 0.51 (open circles; Eq. 47) are shown in Fig. (5B).

$$
\begin{aligned}
& \text { PL 0: } \quad \text { For load } \leq 0.169: \begin{array}{l}
k_{\text {loadTM }}=0.3464+ \\
4.1391 e^{(-7.3778 \text { load })}
\end{array} \\
& \text { For } 0.169<\operatorname{load}<0.5111: \\
& \begin{array}{l}
k_{\text {loadTM }}=1.732- \\
1.1619 \text { load }
\end{array} \\
& \text { For load } \geq 0.5111: \\
& \text { PL 0.51: For load } \leq 0.2396: \begin{array}{l}
k_{\text {loadTM }}=1.2811-0.2797 \text { loadTM } \\
k_{\text {load }}=1.2+ \\
8.2241 e^{(-25.3513 \text { load })}
\end{array} \\
& \text { For } 0.2396<\text { load }<0.47727: \begin{array}{l}
k_{\text {loadTM }}=1.52-(1.4248 \text { load })+ \\
\left(0.7024 \text { load }^{2}\right)
\end{array}
\end{aligned}
$$

For load $\geq 0.47727: \quad k_{\text {loadTM }}=1.0$

For all PLs during relaxation $(\mathrm{V}<-0.5 \mu \mathrm{m} / \mathrm{s})$ : $k_{\text {loadTM }}=1.0$.

To obtain a high velocity for very low load a steeper shortening than expected from the linear $\mathrm{L}_{\min }-\mathrm{F}_{\text {ext }}$ relation was necessary (Fig. 6A). According to the FLR form cardiac muscle the minimum SL is around $1.7 \mu \mathrm{m}$ [33], although smaller SLs were measured in isolated cardiac myocytes [18]. To compromise velocity, shortening in the model was limited to $1.6 \mu \mathrm{m}$. With a change of any other rate parameter $\left(\mathrm{k}_{\text {offI }}, \mathrm{k}_{12}, \mathrm{k}_{3}\right)$ instead of $\mathrm{k}_{\mathrm{tmon}}$, an adjustment to a high velocity at low load was not possible, even with much deeper shortening.

\section{Relaxation Mechanisms}

To speed up isotonic relaxation three mechanisms were considered: SL-dependent titin restoring force, TnI in the "off" conformation pulling Tm to the "off" state and a loaddependent change of $\mathrm{k}_{\text {offI }}$ effective at low load. Titin was shown to play a role during early diastole in the heart [21]. Shortening of the sarcomere below slack length causes the elastic titin spring to be coiled up at the $\mathrm{Z}$ disc [20]. This stored elastic energy is released as restoring force during relaxation and was measured as sarcomere relengthening velocity [21]. The molecular mechanism, how the restoring force acts to turn off muscle contraction, is unknown [21,34]. Here, it was assumed that this force disrupts the structure of the head-to-tail combined Tm molecules (see discussion). During relaxation this is expressed as a faster change of Tm molecules to the "off" conformation for SL below slack length. Independent of the PL for $\mathrm{L}<0.95 \mu \mathrm{m}$ during relaxation $(\mathrm{V}<-0.5 \mu \mathrm{m} / \mathrm{s}$; this value was chosen instead of $\mathrm{V}<0$ for a good model stability.), $\mathrm{k}_{\text {tmoff }}$, the conformational change rate for Tm to the "off" state, (Eq. 10) was multiplied by:

$\operatorname{titinRF}=-16.0\left(L / L_{0}\right)+16.2$

with $\mathrm{L}_{0}$ a normalization factor. The steepness of this equation, i.e., the effect of titin's restoring force, is arbitrary. A linear relationship was chosen, because the SL- relengthening velocity relationship was reported to be linear [21]. Nevertheless, titin's influence is small, since it effects relaxation only if the sarcomere is below slack length.

According to a published model which is based on structural evidence [35], TnI molecules in the "off" conformation pull Tm molecules to the "off" conformation. To account for this cooperativity effect in the present model, during relaxation $\mathrm{k}_{\text {tmoff }}$ (Eq. 10) was increased through the factor $\mathrm{k}_{\text {offTnI }}$ with an increase of the TnI concentration in the "off" conformation, [RUTCaoff], as follows:

$k_{\text {off } T n I}=\left(1+k_{\text {offrelax }}[\text { RUTCaoff }] /[\text { RUtotal }]\right)^{2}$

This change was independent of the applied PL.

At low load for a good late relaxation, a change of the $\mathrm{TnC} \mathrm{Ca}{ }^{2+}$ affinity through an increase in the rate of the TnI conformational change to the "off" conformation $\left(\mathrm{k}_{\mathrm{offI}}\right)$ was essential. During relaxation $\mathrm{k}_{\mathrm{offI}}$ was multiplied by the factor 
$\mathrm{k}_{\text {relaxTn }}$ (Eq. 7), which is load and PL-dependent as depicted in Fig. (5C) (PL0 (filled circles), PL 0.51 (open circles), PL 2.25 (filled triangles)). The fitted equations used for $\mathrm{V}<-0.5$ $\mu \mathrm{m} / \mathrm{s}$ are as follows:

PL 0: For load fraction < 0.338229: $\begin{aligned} & k_{\text {relaxTnI }}=0.5447+ \\ & 3.617 e^{(-6.1125 \text { load })}\end{aligned}$

For load fraction $\geq 0.338229: \quad k_{\text {relaxtnI }}=1.0$

PL 0.51: For load fraction < 0.35795: $k_{\text {relaxnI }}=0.3774+{ }_{(51)}$ $5.5067 e^{(-5.9271 \text { load })}$

For load fraction $\geq 0.35795: \quad k_{\text {relaxinl }}=1.0$

PL 2.25: For load fraction $<0.6427: \quad k_{\text {relax } T h l}=-0.5639+$ $10.2568 e^{(-2.8761 \text { load })}$

For load fraction $\geq 0.6427: \quad k_{\text {relaxThI }}=1.0$

For all PLs during shortening $(\mathrm{V} \geq-0.5 \mu \mathrm{m} / \mathrm{s}): k_{\text {relax } T n l}=1.0$.

\section{Simulations and Data Analysis}

The contraction model was implemented in Java using the simBio package [36], software for cell simulation. The differential equations were solved using an Euler method with dynamically adjusted time steps. The bisection method from "Numerical Recipes" [37] was used to determine L in each time step. The original model is referred to as M1, and the model which lacks the titin function as M2 [14]. In M2 Eq. 12 was exchanged to Eq. 53.

$Q_{M B}=k_{12}[M A D P P i] \times[R U T M o n]_{e f f}-k_{-12}[R U A M A D P P i]$

For steady state simulations a constant $\left[\mathrm{Ca}^{2+}\right]$ was used. Two different ways to obtain a $\mathrm{Ca}^{2+}$ transient were employed. For the twitch contractions (Fig. 2D) and the isotonic contractions (see Fig. 11) the Kyoto Model, a guinea pig based cardiac myocyte model [16], $\mathrm{Ca}^{2+}$ transient was used. For simulations of afterloaded contractions a simple sarcoplasmic reticulum (SR) model [11] was applied. $\mathrm{Q}_{\text {rel }}$ describes the $\mathrm{Ca}^{2+}$ release from the SR and $\mathrm{Q}_{\text {pump }}$ the $\mathrm{Ca}^{2+}$ uptake into the SR.

$$
\begin{aligned}
& Q_{\text {rel }}=Q_{m} \times\left(t / t_{p}\right)^{4} \times e^{4\left(1-t / t_{p}\right)}+Q_{\text {pumprest }} \\
& Q_{\text {pump }}=k_{\text {relaxSR }} \times K_{p} /\left(1+\left(K_{m}\left[\left[\mathrm{Ca}^{2+}\right]^{2}\right)\right.\right.
\end{aligned}
$$

with $K_{m}$ a SR pump parameter, $K_{p}$ the maximum value of $\mathrm{Q}_{\text {pump }}, \mathrm{Q}_{\text {pumprest }}$ the value of $\mathrm{Q}_{\text {pump }}$ under resting $\left[\mathrm{Ca}^{2+}\right]$ conditions, $Q_{m}$ the maximum value of $Q_{\text {rel }}$ and $t_{p}$ the time to $Q_{m}$. For simulations which include the $\mathrm{SR}$ model, the $\mathrm{Ca}^{2+}$ uptake into the SR was enhanced during relaxation at low load expressed in Eq. 56 by the load-dependent factor $\mathrm{k}_{\text {relaxsR. This }}$ load dependent enhancement was necessary due to the lack of $\mathrm{Ca}^{2+}$ buffers others than $\mathrm{TnC}$ in the simple system. In myocytes many $\mathrm{Ca}^{2+}$ buffers operate to avoid sudden $\left[\mathrm{Ca}^{2+}\right]$ changes. Thus, there is no physiological relevance for this load-dependent mechanism. No change was applied to the $\mathrm{Ca}^{2+}$ SR uptake in the Kyoto Model which includes a $\mathrm{Ca}^{2+}$ buffering system [16]. The $\mathrm{k}_{\text {relaxsR-load relationships shown }}$ in Fig. (5C) (PL 0: open triangles; PL 0.51: filled squares; PL 2.25: filled triangles) are as follows:

PL 0: For load fraction < 0.50734: $\begin{aligned} & k_{\text {relaxsR }}=-0.3585+ \\ & 7.6714 e^{(-3.2215 \text { load })}\end{aligned}$

For load fraction $\geq 0.50734: \quad k_{\text {relaxsR }}=1.0$

PL 0.51: For load fraction < 0.59659: $k_{\text {relaxsR }}=-0.4531+$

For load fraction $\geq 0.59659$ :

$7.8531 e^{(-2.7168 \text { load })}$

PL 2.25: For load fraction < 0.6427 :

$k_{\text {relaxsR }}=1.0$

$k_{\text {relas }}=-0.5639+$

$10.2568 e^{(-2.8761 \text { load })}$

For load fraction $\geq 0.6427: \quad \quad k_{\text {relaxsR }}=1.0$

For all PLs during shortening $(\mathrm{V} \geq-0.5 \mu \mathrm{m} / \mathrm{s}): k_{\text {relaxsR }}=1.0$.

For the calculation of the $\left[\mathrm{Ca}^{2+}\right]$ change with time, Eq. 60 was used instead of Eq. 29.

$d\left[\mathrm{Ca}^{2+}\right] / d t=Q_{\text {rel }}-Q_{\text {pump }}-Q_{C a B}$

Mathematical equations used for fitting of the obtained relationships for the load dependence of rate parameters were chosen to give the best possible fit. Mainly equations of the type rational, polynomial or exponential decay were employed. In the case a good fit with the software SigmaPlot (version 10.0; Systat Software, Inc.) with just one equation was not possible, two equations were fixed together at the cutting point, which was determined using MatLab (version 7.3.0.267 (R2006b); The MathWorks, Inc.). The model was quite sensitive concerning the value of load-dependent factors. Therefore, four or more decimal places were required in same cases.

\section{RESULTS}

\section{Regulation of Shortening and Velocity}

The effect of load on the $\mathrm{Xb}$ kinetics during shortening at $37^{\circ} \mathrm{C}$ was studied with simulations of afterloaded isotonic contractions for PL 0, PL 0.51 and PL 2.25 (in $\mathrm{mN} / \mathrm{mm}^{2}$ ). Adjustment of the PL 0 and PL 0.51 models (see Method) to reach the same end-systolic $L\left(L_{\min }\right)$ for the same total load as determined for PL 2.25 (Fig. 6A, M1), resulted in the FVR (Fig. 4B) as expected from experiments (Fig. 4A). Moreover, regardless of the PL the same peak $[\mathrm{Xb}]$ was attained for the same total load (Fig. 6B, M1). Results for the force, $\mathrm{L},[\mathrm{Xb}]$ and velocity time courses for PL 2.25 with various ALs are shown in Fig. (7A), (7B), (7C) and (7D), respectively. For a given PL, the smaller the AL the greater is the amount of shortening, but the faster is the time to $\mathrm{L}_{\min }$ and the higher is the maximum velocity.

A comparison between PL $0,0.51$ and 2.25 for $F=4$ $\mathrm{mN} / \mathrm{mm}^{2}$ is presented in Fig. (8). The different PL models were adjusted to the same $L_{\min }$ for the same force as confirmed in Fig. (8A) and Fig. (8B). The higher the PL the faster was the shortening velocity to reach $\mathrm{L}_{\min }$ (Fig. 8C). The total $[\mathrm{Xb}]$ reaches about the same maximum regardless 

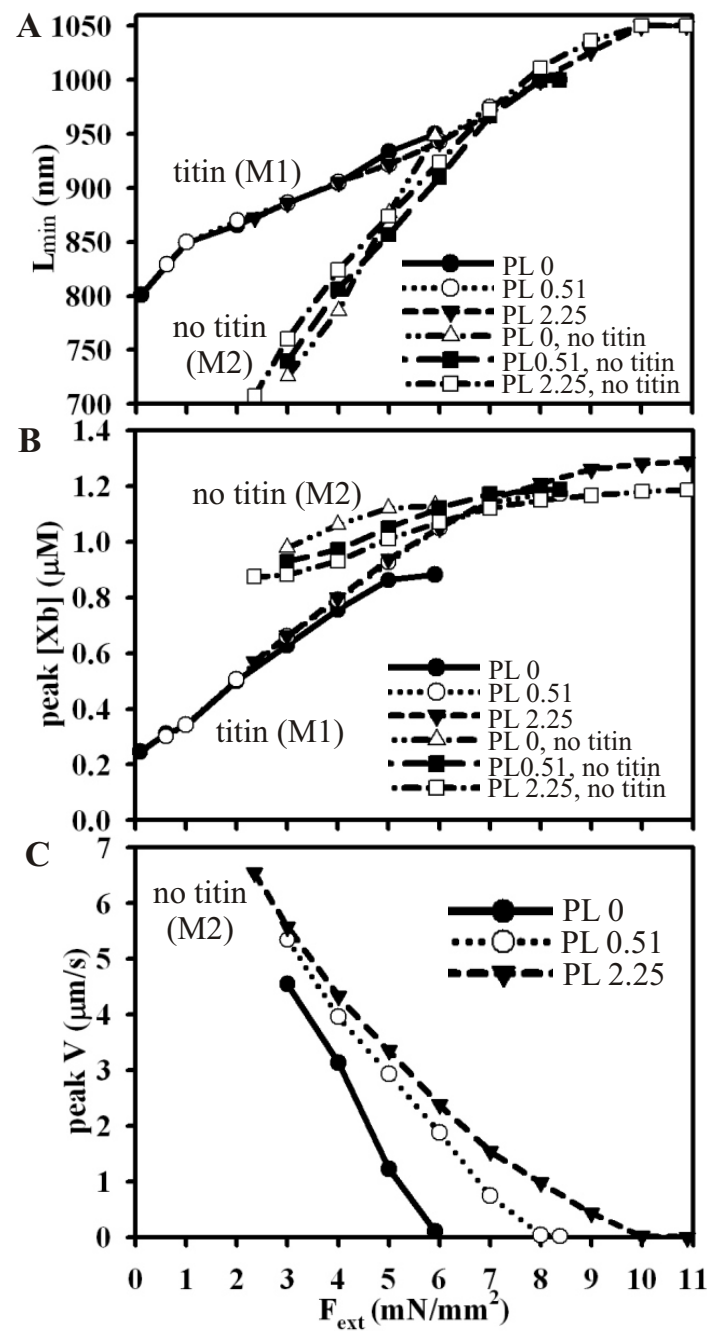

Fig. (6). Simulation results from afterloaded isotonic contractions for $\mathrm{M} 1$ and $\mathrm{M} 2$. In panel $\mathbf{A} \mathrm{L}_{\min }$ and in panel $\mathbf{B}$ the peak $[\mathrm{Xb}]$ are plotted against $\mathrm{F}_{\mathrm{ext}}$ for $\mathrm{M} 1$, the original model with titin-based radial force, and M2, the model which lacks titin, labeled as "no titin”. Panel $\mathbf{C}$ shows the FVR simulation results for M2.

of the PL (Fig. 8D). Since for the same $\mathrm{L}_{\min } \mathrm{F}_{\mathrm{p}}$ is the same, $F_{b}$ has to be the same to satisfy Eq. 31. The small difference of the peak $[\mathrm{Xb}]$ is due to the $(\mathrm{L}-\mathrm{X})$ term in Eq. 30. The minimum of $(\mathrm{L}-\mathrm{X})$ coincides with the peak of the $[\mathrm{Xb}]$ (Fig. 8E) and the peak velocity (Fig. 8F). The higher the value of the $(\mathrm{L}-\mathrm{X})$ minimum, the less $\mathrm{Xbs}$ are necessary to get to the same $F_{b}$, i.e., the more force has the average $\mathrm{Xb}$ (Fig. 8D and 8G). With an increase in the shortening velocity, the $\mathrm{Xb}$ force decreases (Fig. 8H), i.e., with an increase in load the average $\mathrm{Xb}$ force increases (insert in Fig. 8H). For PL $0 \mathrm{~L}_{\min }$ was reached with a delay (Fig. 8B). Due to the short SL the $\mathrm{Xb}$-induced cooperative activation was reduced (smaller KTitin, Eq. 16), which resulted in a slower activation of RUs (RUTMon) and a smaller fraction of effective RUTMon. Although the load-dependent change of the $\mathrm{Xb}$ force is small compared to the change of the $[\mathrm{Xb}]$, it is essential, since a model with a constant (L-X), i.e., $F_{b}$ only dependent on $[\mathrm{Xb}]$ was instable and could not be adjusted to give the desired FVR (data not shown).

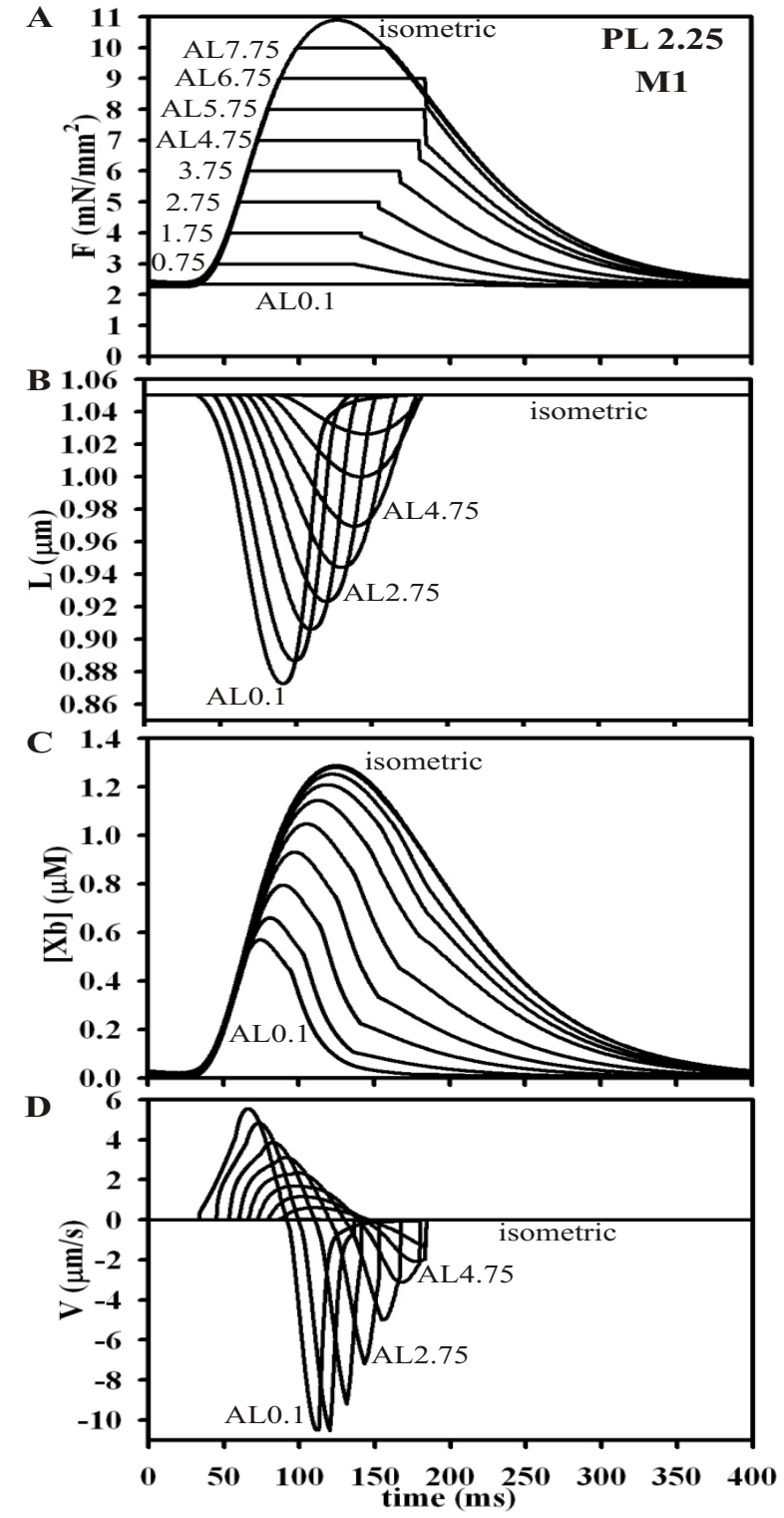

Fig. (7). Simulation results from afterloaded isotonic contraction with PL 2.25. For various ALs the time courses for force (A), L $(\mathbf{B}),[\mathrm{Xb}](\mathbf{C})$ and the shortening and relaxation velocity (D) are shown. The model which includes all relaxation mechanisms was used for the simulations.

In conclusion, the ADP release rate controls the peak [Xb] at high to medium load, the physiological working range of the heart, which corresponds to a velocity of up to about 5-6 $\mu \mathrm{m} / \mathrm{s}$ in the model (Fig. 9A). The peak $[\mathrm{Xb}] \mathrm{de}-$ creases linearly with velocity in this range (Fig. 9A) as expected form a linear rise of $\mathrm{k}_{5}$ with velocity (Fig. 9B). But at higher velocity a common value of about $0.3 \mu \mathrm{M}$ is reached independent of the PL, suggesting that a physiological minimum is attained. The minimum $[\mathrm{Xb}]$ is maintained through a faster activation of RUs achieved through an increase in the Tm conformational change rate mediated by the factor $\mathrm{k}_{\text {loadTM }}$ for velocities greater than $5 \mu \mathrm{m} / \mathrm{s}$ (Fig. 9B). The $\mathrm{Xb}$ force, expressed by (L-X), decreases linearly with a 

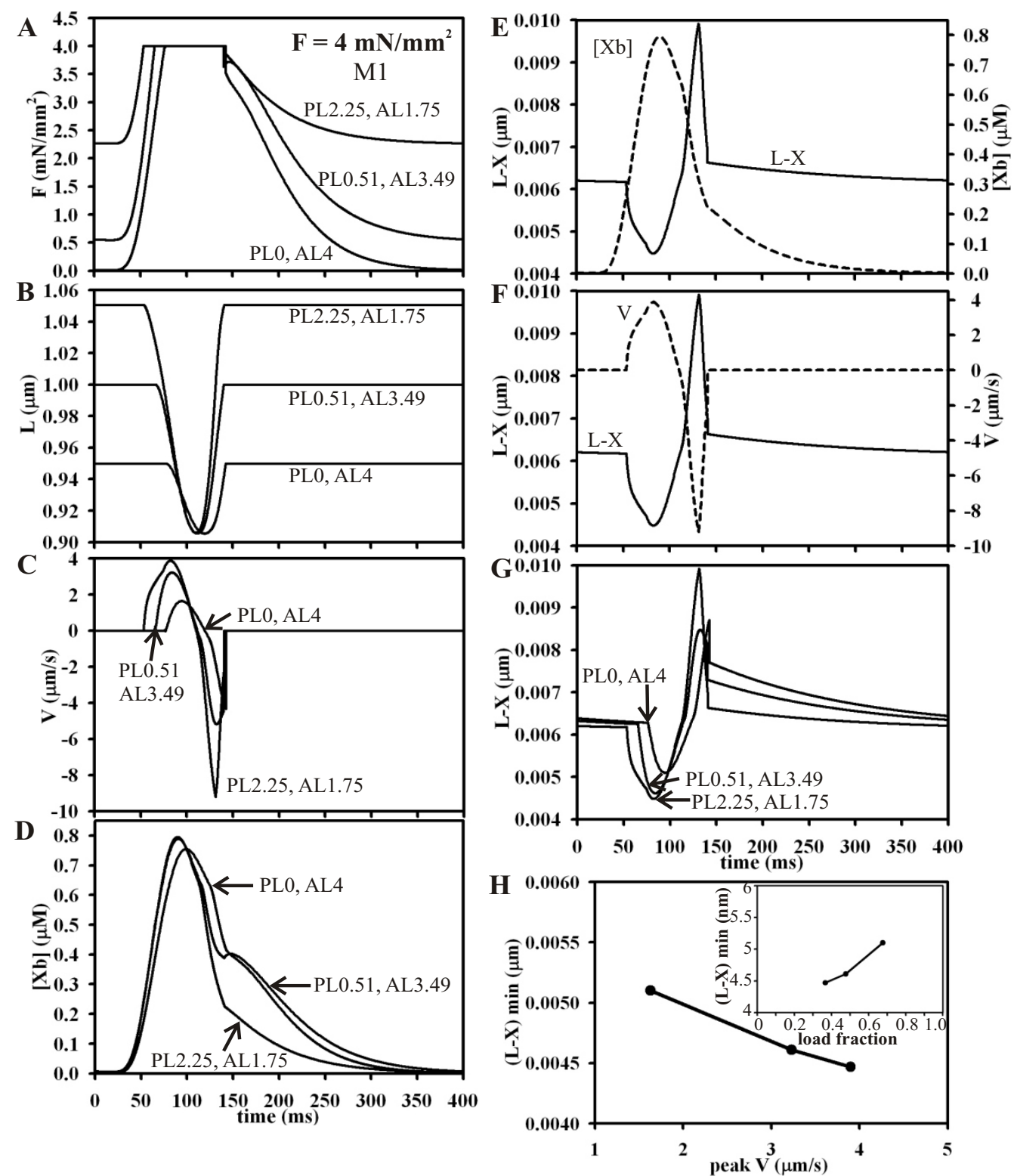

Fig. (8). Comparison of afterloaded isotonic contractions obtained with different PL models reaching the same total load $\mathrm{F}=4 \mathrm{mN} / \mathrm{mm}^{2}$. The time courses for force $(\mathbf{A}), \mathrm{L}(\mathbf{B})$, velocity $(\mathbf{C})$ and $[\mathrm{Xb}](\mathbf{D})$ are shown for the following load combinations: PL 0 with AL 4, PL 0.51 with AL 3.49 and PL 2.25 with AL 1.75. The minimum of (L-X) coincides with the peak [Xb] (E) and the maximum shortening velocity $(\mathbf{F})$. Panel $\mathbf{G}$ shows the (L-X) time courses for the three load combinations. The (L-X) minima for the three load combinations are plotted against the peak velocity $(\mathbf{H})$ or against the load fraction (insert in $\mathbf{H})$.

rise in velocity (Fig. 9A), which is independent of the PL. However, this change is small compared to the change of $[\mathrm{Xb}]$, suggesting that the FVR is mainly determined by the [Xb] (Fig. 8C, 8G and 8H).

\section{The Influence of Titin-Based Radial Force on Shortening and Velocity}

To elucidate the effect of titin-based radial force on shortening and velocity, the SL-dependent influence of titin on the myosin binding rate was eliminated (M2), with no changes of the $\mathrm{k}_{5}$ and $\mathrm{k}_{\mathrm{loadTM}}-$ load relationships. Simulation results for PL $0,0.51$ and 2.25 are shown in Fig. (6). Although at high $\mathrm{F}_{\text {ext }} \mathrm{L}_{\min }$ obtained with M2 is similar to the
$\mathrm{L}_{\min }$ reached with the titin model (M1), for smaller loads a much smaller $\mathrm{L}_{\min }$ compared to M1 was found (Fig. 6A). The peak $\left[\mathrm{Xb}\right.$ ] change with $\mathrm{F}_{\mathrm{ext}}$ was very small for M2 (range: $0.875 \mu \mathrm{M}$ to $1.186 \mu \mathrm{M}$ for PL 2.25) compared to M1 (range: 0.57 to $1.288 \mu \mathrm{M}$ ) (Fig. 6B). Due to the large shortening amount at smaller $F_{\text {ext }}$, the peak velocity is higher for M2 (Fig. 6C) compared to M1 (Fig. 4B). Our simulation results predict that titin-based radial force variations with SL prevent cardiac muscle to reach smaller SLs at medium to low load.

Fig. (10A) shows the PL 0.51 force time courses from afterloaded isotonic contractions for M1 and Fig. (10B) for M2. In the medium load range a decrease in AL was accom- 


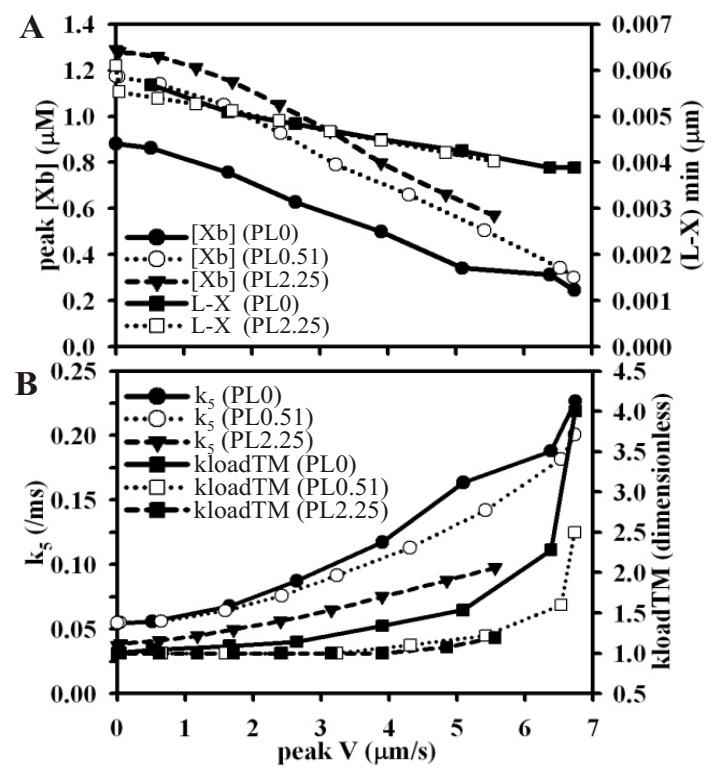

Fig. (9). The dependence of the peak $[\mathrm{Xb}]$ and the ADP release rate on the velocity. In panel $\mathbf{A}$ the peak $[\mathrm{Xb}]$ and $(\mathrm{L}-\mathrm{X})$ from afterloaded contractions obtained with the PL 0 and 2.25 models and in panel $\mathbf{B} \mathrm{k}_{5}$ and $\mathrm{k}_{\text {loadTM }}$ are plotted against the velocity.

panied by a faster start of the isometric relaxation in M1, but almost no change was found in results obtained with M2. The AL-dependent onset change of the isometric relaxation can be attributed to the shortening-induced cooperative deactivation in M1 which is missing in M2. Due to the SLdependence of the myosin binding rate $\left(\mathrm{k}_{12}\right)$ caused by titin's radial force in $\mathrm{M} 1$, sarcomere shortening slows down $\mathrm{k}_{12}$ and therefore strong $\mathrm{Xb}$ formation, which in turn reduces thin filament activation via an increase in $\mathrm{k}_{\text {offI }}$ (Fig. 1A) [14]. The smaller the AL the smaller is $\mathrm{L}_{\min }$ and the faster is the deactivation. This deactivation reduces the shortening velocity in M1 as compared to M2 (inserts in Fig. 10A and 10B).

\section{Analysis of Relaxation Mechanisms}

Titin-based radial force in M1 prevents a fast isotonic relaxation speed (insert in Fig. 10A), since an increase in the $\mathrm{SL}$ promotes activation and delays force relaxation. This $\mathrm{Xb}-$ induced cooperative activation is missing in M2 (insert in Fig. 10B). To improve relaxation, which was reported to be faster than shortening [38], three mechanisms were considered (see Method): 1. a SL-dependent effect from titin restoring force, 2. TnI in the "off" conformation pulling Tm to the "off" state and 3. a load-dependent change of $\mathrm{k}_{\text {off }}$ only effective at low load.

Fig. (10C) exhibits force time course data for various ALs from simulations obtained with the PL 0.51 M1 that includes the above relaxation mechanisms. A slightly faster relaxation than shortening was achieved (insert in Fig. 10C). The time interval from stimulation to the onset of isometric relaxation $(\mathrm{RST}=$ relaxation start time) was chosen for model adjustment. A comparison of simulation data from relaxation-adjusted and unadjusted models with experimental data is shown in Fig. (10D). Except for the steepness of the slope, simulation data are comparable to experimental data from cat. To achieve a faster velocity for relaxation than for shortening, the steeper relationship was necessary for the model. However, the steepness might depend on various factors such as temperature and stimulation frequency. As found in experiments, simulation results show a decrease in RST at very high load and an increase at very low load (Fig. $10 D$ and 10E). For high ALs relaxation could be adjusted using only titin restoring force (Eq. 48) and the [RUTCaoff] cooperativity feedback mechanism (Eq. 49) as shown in Fig. (10F). As demonstrated in Fig. (10E), regardless of the PL, for the same $F_{\text {ext }}$ the same RST was found. For lower loads, as indicated by arrows, a load-dependent increase of $\mathrm{k}_{\text {offI }}$ (Eq. 50-52) was necessary. The higher the PL the stronger was this load dependence of the relaxation. The steepness of the slope is due to the strength of the titin restoring force and the pulling force TnI exerts on Tm. To show how each mechanism influences relaxation, simulations with modified models for PL 0.51 and AL 0.49 were performed. As depicted in Fig. (10G) (line with small dash) titin restoring force alone only speeds up the very early relaxation, whereas the [RUTCaoff] cooperativity feedback is more powerful in effecting the early relaxation (dash with two dots). However, at this low load for a fast late relaxation an increase of $\mathrm{k}_{\text {off }}$ was essential. A faster $\mathrm{k}_{\text {off }}$ rate indirectly enhances the $\mathrm{Ca}^{2+}$ release from $\mathrm{TnC}$. If the $\mathrm{Ca}^{2+}$ transient was produced by a simple SR model without $\mathrm{Ca}^{2+}$ buffers, bumps in the $\mathrm{Ca}^{2+}$ transient occurred (Fig. 10H). In this case, an enhanced SR $\mathrm{Ca}^{2+}$ uptake was necessary at lower load to achieve full relaxation (Eq. 57-59). No bumps were observed if the myocyte model was employed due to $\mathrm{Ca}^{2+}$ buffering.

\section{Model Validation- Isotonic Contractions in a Myocyte Model}

With the Kyoto Model [16], which produces a more physiological $\mathrm{Ca}^{2+}$ transient, only simulations of nonafterloaded $(\mathrm{AL}=0)$ contractions are possible. Recently, it was reported that with an increase in PL the peak $\left[\mathrm{Ca}^{2+}\right]$ increases in a range from $0.8 \pm 0.1 \mu \mathrm{M}$ for PL 0 to $1.45 \pm 0.25$ $\mu \mathrm{M}$ for optimal $\mathrm{SL}$ in rabbit trabeculae $\left(37^{\circ} \mathrm{C}, 2 \mathrm{~Hz}, 2.5 \mathrm{mM}\right.$ $\left[\mathrm{Ca}^{2+}\right]_{\mathrm{o}}$ ) [39]. In the present model adjustment of the peak $\left[\mathrm{Ca}^{2+}\right]$ through a change of the L-type Calcium channel $\mathrm{Ca}^{2+}$ permeability [16] was found to be important to obtain a shortening amount which is comparable to experimental data. Fig. (11A) shows the simulation results for the isotonic shortening time courses for different PLs compared to experimental data. The shortening amount changes with PL as follows: PL 0, 8.2\%; PL 0.51, 10.0\%; PL 2.25, 11.9\%. This is in the range found experimentally for guinea pig myocytes $[18,40]$. The $\mathrm{Ca}^{2+}$ transients are depicted in Fig. (11B). The normalized $\mathrm{Ca}^{2+}$ transients are very similar suggesting no influence in the relaxation (insert in Fig. 11B) as reported from experiments [39]. The velocity time courses are depicted in Fig. (11C) showing that relaxation velocities are faster than shortening velocities.

\section{DISCUSSION}

The present study stands out among other simulation studies performed to analyze the regulation of shortening and relaxation because of the unique cardiac muscle contraction 

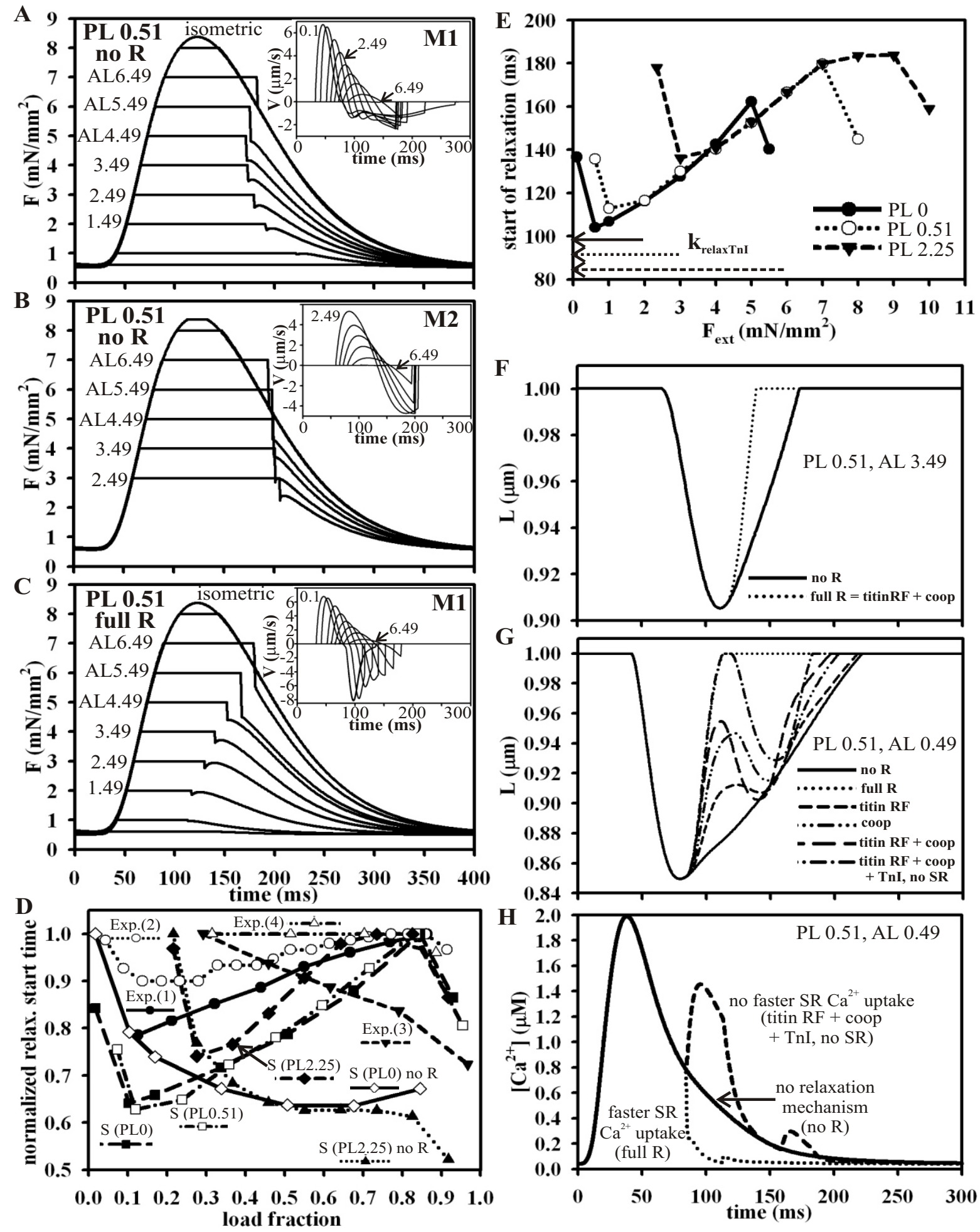

Fig. (10). Simulation analysis of shortening-induced deactivation and relaxation. The force time courses from afterloaded isotonic contraction for PL 0.51 are shown for M1 (A) and M2 (B) before adjustment of relaxation, i.e., without Eq. 48, Eq. 49, Eq. 51 and for M1 after adjustment of relaxation $(\mathbf{C})$. The inserts in $\mathbf{A}, \mathbf{B}$ and $\mathbf{C}$ depict the velocity time courses. Shortening-induced deactivation is absent in M2 (B). Panel D depicts the onset of the isometric relaxation of afterloaded isotonic contractions, which was measured from the start of the stimulation and normalized to the maximum of a data set, plotted against the load fraction for the following experimental data (Exp.) and simulations (S): Exp. (1), cat cardiac muscle, $29^{\circ} \mathrm{C}$ [51]; Exp. (2), cat cardiac muscle, $26^{\circ} \mathrm{C}$ [30]; Exp. (3), rat cardiac muscle, 29 ${ }^{\circ} \mathrm{C}$ [51]; Exp. (4), rat cardiac muscle, $30^{\circ} \mathrm{C} \quad$ [52]; $\mathrm{S}$ (PL 0), $\mathrm{S}$ (PL 0.51) and $\mathrm{S}$ (PL 2.25), simulation with the PL model with full relaxation 
Fig. (10). Contd....

(Eq. 48-52); S (PL 0) no R and S (PL 2.25) no R, simulation with the PL model without adjustment of relaxation. In panel $\mathbf{E}$ the start time of the isometric relaxation given in (ms) for the three PL models with full relaxation is plotted against $\mathrm{F}_{\text {ext }}$. The arrows indicate for which loads the relaxation was influenced by $\mathrm{k}_{\text {relaxTnI }}$ (Eq. 50 for PL 0, Eq. 51 for PL 0.51 and Eq. 52 for PL 2.25). Panel $\mathbf{F}$ exhibits the L time courses for PL 0.51/ AL 3.49 (higher load) for two PL 0.51 model variants: no R, no relaxation (M1 without Eq. 48, Eq. 49, Eq. 51 and Eq. 58) and full R, full relaxation (M1 with Eq. 48, Eq. 49, Eq. 51 and Eq. 58). At this load $\mathrm{k}_{\text {relaxTnI }}=\mathrm{k}_{\text {relaxSR }}=1$ and therefore full $\mathrm{R}=$ titin $\mathrm{RF}+$ coop, i.e., relaxation is load independent. Panel $\mathbf{G}$ shows the L time courses for PL 0.51/ AL 0.49 (low load) obtained with different PL 0.51 model variants as follows: no R, no relaxation (M1 without Eq. 48, Eq. 49, Eq. 51 and Eq. 58); full R, full relaxation (M1 with Eq. 48, Eq. 49, Eq. 51 and Eq. 58); titin RF, M1 with Eq. 48, lacks Eq. 49, Eq. 51 and Eq. 58; coop, M1 with Eq. 49, lacks Eq. 48, Eq. 51 and Eq. 58; titin RF+coop, M1 with Eq. 48, Eq. 49, lacks Eq. 51 and Eq. 58; titin RF+coop+TnI, no SR, M1 with Eq. 48, Eq. 49 and Eq. 51 , lacks Eq. 58. Panel $\mathbf{H}$ shows the $\mathrm{Ca}^{2+}$ transient from some model variants as described for panel $\mathbf{G}$.

model used. Although mathematically simple, it is biologically one of the most advanced models. In addition to having

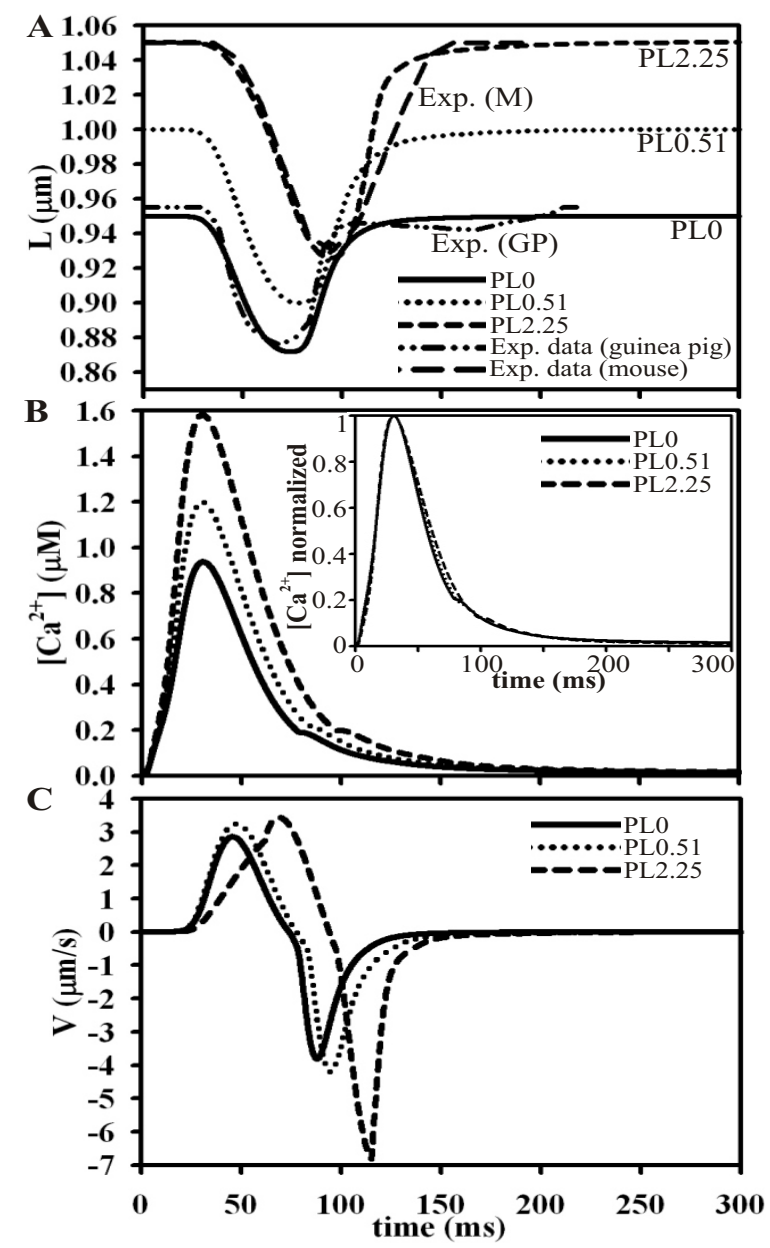

Fig. (11). Simulations of isotonic contractions with the Kyoto Model $\mathrm{Ca}^{2+}$ transient. In panel $\mathbf{A}$ simulation results for the $\mathrm{L}$ time courses for PL $0,0.51$ and 2.25 are compared to experimental data from a guinea pig left ventricular myocyte labeled as Exp. (GP) $\left(37^{\circ} \mathrm{C}\right.$, slack length) $[18]$ and a mouse right ventricular trabecula labeled as Exp. (M) (SL 2 to $2.1 \mu \mathrm{m}, 37^{\circ} \mathrm{C}$ ) [61]. The $\mathrm{Ca}^{2+}$ transients for the three PLs are shown in panel $\mathbf{B}$. The peak of the $\mathrm{Ca}^{2+}$ transients were adjusted for the different PLs through a change of the Ca permeability of the L-type calcium channel $\left(\mathrm{P}_{\mathrm{CaL}}\right.$ of $\mathrm{I}_{\mathrm{CaL}}$ in the Kyoto Model; Table 4, Eq. 19 in [16]) as follows: $\mathrm{PCaL}=4700$ for PL 0, 6000 for PL 0.51 and 8000 for PL 2.25. The insert shows the normalized $\mathrm{Ca}^{2+}$ transients. Panel $\mathbf{C}$ depicts the velocity time courses for the three different PLs. physiological defined states, which stands in contrast to many previous models including a recently published model [12], various mechanisms such as a potential Frank-Starling mechanism, which was lately supported by some experimental evidence [41], and different cooperativity mechanisms [14] are included. Six out of 15 rate parameters are dynamically changing with species concentrations, SL or load, representing a quite realistic biological situation, where key reactions are constantly regulated to maintain homeostasis as e.g., the metabolic homeostasis of the heart [42]. Moreover, the present model includes detailed thin filament activation and is the only model that considers the conformational change of TnI, which was found to be an important regulatory step [43]. Rather than presenting normalized simulation results, as generally done in published contraction models, biological relevant data are given. This biological model structure can support the discovery of unknown mechanisms. Furthermore, this is the first simulation study that analyzes afterloaded contractions for different PLs in a quantitative way. Suggested mechanisms and simulation results are discussed below in context of model assumptions and limitations and compared to experimental findings.

\section{The Rate Limiting Step in Myocyte Shortening}

Simulation results highly depend on the chosen values for rate parameters in addition to model structure, since this determines the rate limiting steps, and further rely on which steps are decided to be load, SL or cooperativity dependent. Experimental evidence suggests that the ADP release rate is load-dependent [44-47] rather than the $\mathrm{P}_{\mathrm{i}}$ release [45]. But which step of the $\mathrm{Xb}$ cycle is rate limiting is still not clarified. Based on skeletal muscle experiments the ADP release rate was reported to be the limiting step $[44,46,47]$. In contrast, Hinken \& McDonald [48] showed using skinned rat cardiac myocytes at $13^{\circ} \mathrm{C}$, that addition of $\mathrm{P}_{\mathrm{i}}$ decreased force, but increased loaded shortening velocity for loads greater than $10 \%$ of the isometric force. They concluded that the $\mathrm{P}_{\mathrm{i}}$ release is the rate limiting step at medium to higher load. Accordingly, the rate limiting step may well be changing with load. But results may also depend on the temperature and on temperature dependencies of rate parameters. In the present study, the ADP release rate $\left(k_{5}\right)$ was chosen for model adjustment because it was the rate limiting step in the $\mathrm{Xb}$ cycle under isometric conditions and therefore appropriate for a key regulatory step. The model could well be adjusted for the desired velocity and end-systolic SL for medium to high load solely with a load-dependent change of $k_{5}$. 
The rise of $k_{5}$ is linear with an increase in velocity (Fig. 9B). At low load, however, the $P_{i}$ release rate becomes rate limiting in the $\mathrm{Xb}$ cycle. A high velocity at low load could only be achieved with a high $\mathrm{Xb}$ turnover through an accelerated $\mathrm{Tm}$ conformational change mediated by the factor $\mathrm{k}_{\text {loadTM }}$ together with an increase in $k_{5}$. At low to medium velocity $\mathrm{k}_{\text {loadTM }}$ is constant, but increases exponentially at high velocity (Fig. 9B). These rate parameter changes with velocity are comparable to experimental results from frog skeletal muscle [49]. A linear rise of the detachment rate with an increase in velocity was reported. The calculated apparent attachment rate under assumption of a two-state model was non-linearly increasing with velocity.

In the present model, the Tm conformational change rate at low load is the rate limiting step in the thin filament activation and its change was found to be most effective in obtaining a high velocity. So far this step has not been shown to be load or velocity dependent.

\section{Regulation of Shortening- The [Xb] Determines the End- Systolic Sarcomere Length}

Simulation data revealed that at medium to high load, which corresponds to a shortening velocity smaller than about 5 to $6 \mu \mathrm{m} / \mathrm{s}$, the amount of shortening is determined by the peak $[\mathrm{Xb}]$, which decreases linearly with velocity (Fig. 9A). A small variation of the peak $[\mathrm{Xb}]$ is due to the fact that the $\mathrm{Xb}$ force is slightly velocity dependent (Fig. 8D and $\mathbf{8 G}$ ). At low load, regardless of the PL, the peak $[\mathrm{Xb}]$ reaches the same almost constant value, and velocity is regulated by the thin filament activation speed (Fig. 9). Recently, X-ray interference and mechanical measurements performed with frog skeletal muscle fibers at $4^{\circ} \mathrm{C}$ revealed a linear decrease of attached Xbs with an increase in velocity [49]. Furthermore, these experiments showed that the force per attached $\mathrm{Xb}$ decreased with increasing velocity. But for small velocities these changes were small compared to the $[\mathrm{Xb}]$ change. From these data it was concluded that the FVR results from a decrease of the [Xb] with a fall in load [49]. Our simulation results coincide well with the above findings (Fig. 9). This is in contrast to previous suggestions, that the $\mathrm{Xb}$ force highly depends on load [6,7]. Comparing cardiac muscle simulation data with results form skeletal muscle experiments implies, however, that the chemomechanical transductions are at least similar between different myosins, an assumption that might be justified [47].

Although the present model contains a complex kinetics, the underlying mechanical principles are very simple. In particular, the ADP detachment rate depends on load, but is not directly influenced by the movement and strain of the $\mathrm{Xb}$. Thus, the model only shows how the value of the rate parameters need to be modified with load, but cannot predict how the change is controlled by Xbs. So far these mechanisms have not been revealed by experiments making assumptions unavoidable in modeling. Many models specifically focusing on the mechanics were developed $[6,9,12]$. Various models utilize a variation of the Huxley-type model [7] with the detachment rate depending on the distance between the actin binding site and the myosin equilibrium posi- tion $[6,12]$. In a recent Huxley-type model by Negroni and Lascano (NL model) [12] two attached $\mathrm{Xb}$ states, named weak and power state, were postulated each having a different elongation and therefore force. As in the present model the same basic $\mathrm{Xb}$ mechanics equation (Eq. 32) was used in the NL model but with a different interpretation and values of the parameters. The $\mathrm{Xb}$ detachment in the present model is coupled with the ADP release, and the detachment rate parameter $\left(\mathrm{k}_{5}\right)$ is load-dependent. In contrast, in the NL model, which does not specify the $\mathrm{P}_{\mathrm{i}}$ or ADP release steps, the detachment occurs from a weak state. The detachment rate parameter depends on SL and the elongation of the weak $\mathrm{Xb}$ state, which is velocity dependent. Whereas in the present study B (Eq. 32) was shown to represent a loaddependent myosin characteristic which determines the shortening velocity in phase 2 of force step experiments (Eq. 39; Fig. 3C), in the NL model B is a constant. However, the NL model fails to correctly reproduce phase 2 of force step experiments with a not realistic length of the myosin stroke (Fig. 8A in [12]). These Huxley-type models have in common that the $\mathrm{Xb}$ force is greatly reduced during shortening and the myosin stroke size depends on the shortening velocity. However, recent experimental data challenged that view [49]. The stroke size was reported to be more or less constant over a large velocity range. Only a small change of the $\mathrm{Xb}$ force was detected at small to medium velocities and the $[\mathrm{Xb}]$-velocity relationship was linear. The present model with just a load-dependent ADP release rate and both strong $\mathrm{Xb}$ states having the same constant force successfully reproduced these findings (Fig. 9).

Adjustment of the isometric model for the maximum [Xb] was done for full activation, i.e., tetanic steady state at high $\left[\mathrm{Ca}^{2+}\right]$ and maximum SL $(2.2 \mu \mathrm{m})$ to match experimental data performed under similar conditions (see section 3.1. in [14]). However, the degree and speed of the thin filament activation had to be balanced with the time to peak isometric force. For isotonic contractions especially at higher load and smaller SL the obtained number of Xbs is lower than physiologically supposed. Although this needs to be improved in the future, it is not expected to influence the current conclusions.

\section{Titin-based Radial Force Mediates Shortening-Induced Cooperative Deactivation}

The giant titin molecule has been implicated in many functions, due to its length and different domains [34]. While titin has no direct role in the actin-myosin interactions during shortening, it exhibits various regulatory roles. Although still controversial [34], titin-based radial force may alter the interfilament spacing in a SL-dependent manner. This mechanism was included in the present model and tested for isometric contractions in a previous study [14]. A steep FLR as observed for cardiac muscle could be achieved with a SLdependent change of the myosin binding rate. A lack of this titin-based mechanism caused a typical skeletal muscle FLR in the isometric model [14]. In the isotonic model, already at medium load a steep shortening to small SLs was observed (Fig. 6A). Such SLs are not naturally found in the intact 
heart [50]. In the model SL shortening results in a titin regulated increase of the lateral space between thin and thick filaments, which slows down strong $\mathrm{Xb}$ formation. A decrease in the $[\mathrm{Xb}]$ enhances the rate for the TnI conformational change to the "off" state, thereby deactivating thin filaments. This potential Frank-Starling mechanism [14] accounts in the model for the shortening-induced cooperative deactivation. With a decrease of $\mathrm{AL}$ from a high to medium value, the time isometric relaxation starts is decreased in the model with titin (M1, Fig. 10A), but not in the model without titin (M2, Fig. 10B). Such load dependence was reported for afterloaded isotonic contractions performed with cat and pig cardiac muscle, but found to be small for rat and was missing in frog [30,51]. Furthermore, a decrease of this effect with an increase in temperature was detected in rat myocardium [52]. These species differences were suggested to depend on the $\mathrm{Ca}^{2+}$ uptake by the SR [30,51]. However, as predicted here, they may be explained by the included FrankStarling mechanism. A variation of the titin isoform ratio, which is known to be highly species and tissue dependent [53], results in a varying strength of titin-based radial force. This may cause the different load dependence. Experimental evidence that strong Xbs modulate shortening velocity was reported: Length steps performed with rat single skinned cardiac myocytes revealed a progressive slow down of the shortening velocity at submaximum $\left[\mathrm{Ca}^{2+}\right]$, which could be reduced by addition of $\mathrm{N}$-ethylmaleimide-conjugated myosin subfragment-1 acting as a strong $\mathrm{Xb}$ equivalent [54]. A full mechanism, as proposed here, has not been revealed before. However, sarcomere shortening, resulting in an increase in filament spacing and hence, in a decrease of $\mathrm{Xb}$ formation, was proposed as part of the shortening-induced cooperative deactivation mechanism, stated to be important for relaxation as "myofibrillar brake system" [15]. Our simulation results show that this mechanism is significant, but alone cannot account for a fast and complete relaxation, especially at lower load (Fig. 10A).

\section{Fast Relaxation due to Enhanced Thin Filament Deacti- vation}

An altered ventricular relaxation results in an impaired filling and therefore an inappropriate cardiac output. Since diastolic dysfunction is associated with various cardiomyopathies, elucidating the mechanisms underlying relaxation is of great importance [55]. Isotonic relaxation was shown to be faster than isotonic shortening [38]. Although various intrinsic and extrinsic mechanisms were found to play a role in the regulation [55], how cardiac muscle achieves these high relaxation velocities is not well understood. The fast early relaxation phase was recently attributed to titin restoring force [21] and could be explained as elastic recoil of the titin molecule [20]. However, how the release of the elastic energy from each single titin molecule speeds up relaxation is unknown. Experiments show no change of the interfilament lattice spacing, but a conformational disorder was suggested around the start of relaxation since a buckling of myosin filaments was observed [21]. Here, it was assumed that the detected disorder might be a disruption of the Tm filament structure leading to a SL-dependent increase in the Tm conformational change rate to the "off" state $\left(\mathrm{k}_{\text {tmoff }}\right)$ during relaxation. A variation of $\mathrm{k}_{\text {tmoff }}$ greatly affected relaxation speed, whereas a variation of $\mathrm{Xb}$ cycle rate parameters such as an increase in $\mathrm{k}_{-12}, \mathrm{k}_{-3}, \mathrm{k}_{-4}$ or $\mathrm{k}_{5}$ had only a minor effect. While an increase in $\left[\mathrm{P}_{\mathrm{i}}\right]$ was reported to speed up relaxation [56], in the model a faster $\mathrm{P}_{\mathrm{i}}$ addition did not improve relaxation. Moreover, an increase in the transition from strong Xbs to pre-power states during stretch was suggested to save chemical energy and enhance relaxation [56]. The question remains what prevents these pre-power states from reattachment. This of course occurs in the present model preventing a fast relaxation. Overall in the model the effect from titin restoring force was rather small (Fig. 10G) suggesting that this is not a major relaxation mechanism.

A structural study revealed that TnI in the "off" conformation pulls Tm to the "off" conformation [35]. This mechanism was further supported by an electron microscopy study that showed that at a low $\left[\mathrm{Ca}^{2+}\right]$ the $\mathrm{C}$-terminal $\mathrm{TnI}$ domain competes with $\mathrm{Tm}$ for a common actin binding site, thus pushing $\mathrm{Tm}$ into the blocking position [57]. Recently, the TnI Lys184 deletion, which is located in the C-terminal mobile domain and linked to familial hypertrophic cardiomyopathy, which is associated with diastolic dysfunction, was reported to slow down relaxation in mouse leftventricular papillary muscle fibers and to increase thin filament $\mathrm{Ca}^{2+}$ sensitivity [58]. In our model a feedback of [RUTCaoff] on $\mathrm{k}_{\text {tmoff }}$ strongly speeded up early relaxation (Fig. 10G). This mechanism together with the titin restoring force was sufficient for a fast relaxation velocity at medium to high load (Fig. 10E and 10F).

With decreasing load, late relaxation was slowed, especially at higher PLs (Fig. 10E-G), but could be improved through a load-dependent increase in the TnI conformational change rate to the "off" state $\left(\mathrm{k}_{\text {offI }}\right)$. An increase of $\mathrm{k}_{\text {off }}$ during relaxation might play an important role in the extrinsic regulation of cardiac output. TnI phosphorylation by protein kinase $A$ during $\beta$-adrenergic stimulation weakens the $\mathrm{TnC}$ $\mathrm{Ca}^{2+}$ affinity and enhances relaxation [59]. Furthermore, experiments showed an increase in $\mathrm{TnI}$ phosphorylation with an increase in AL [60].

\section{CONCLUSION}

The development of a unique cardiac muscle contraction model that includes detailed thin filament activation is described here. $\mathrm{Xb}$ mechanics was adjusted through simulations of $\mathrm{P} 1$ and $\mathrm{P} 2$ of force steps. Adjustment of the $\mathrm{Xb}$ kinetics was achieved through simulations of afterloaded isotonic contractions, while considering that for different PLs but the same total load, the same end-systolic SL is reached. Simulation results show that in the load range relevant to the intact heart the end-systolic SL was mainly determined by the $[\mathrm{Xb}]$, while the $[\mathrm{Xb}]$ was regulated by the loaddependent ADP release rate. Instead, the change of the $\mathrm{Xb}$ force with a change in the shortening velocity was small. Shortening-induced cooperative deactivation was attributed to the included Frank-Starling mechanism. For a good relaxation the speed of the thin filament deactivation was more significant than the $\mathrm{Xb}$ detachment rate. A fast relaxation 
was achieved mainly through an increased rate of the Tm conformational change to the "off" state.

In the future, this model will need to be tested for its energetic performance and for auxotonic contractions in a circulation model to mimic the intact heart conditions with PL and AL constantly changing. Due to its biological realism the model, which can be easily inserted into any myocyte or circulation model, will allow for significantly more realistic physiological and pathophysiological cardiac muscle contraction studies than previously available models.

\section{ACKNOWLEDGEMENTS}

The authors wish to thank Prof. Matsuoka for suggestions to improve this manuscript. The first author expresses her gratitude to Prof. Mitsuiye for providing an office space. This study was supported by the Leading Project for Biosimulation from the Ministry of Education, Culture, Sports, Science and Technology of Japan.

\section{ABBREVIATIONS}

$\begin{array}{ll}\mathrm{AL} & =\text { Afterload } \\ \mathrm{FVR} & =\text { Force-velocity relationship } \\ \mathrm{L}_{\min } & =\text { End-systolic } \mathrm{L} \\ \mathrm{LTR} & =\text { Length-tension relationship } \\ \mathrm{MHC} & =\text { Myosin heavy chain } \\ \mathrm{P} 1 & =\text { Phase } 1 \\ \mathrm{P} 2 & =\text { Phase } 2 \\ \mathrm{PL} & =\text { Preload } \\ \mathrm{RU} & =\text { Regulatory unit } \\ \mathrm{SL} & =\text { Sarcomere length } \\ \mathrm{SR} & =\text { Sarcoplasmic reticulum } \\ \mathrm{Tm} & =\text { Tropomyosin } \\ \mathrm{Tn} & =\text { Troponin } \\ \mathrm{TnC} & =\text { Troponin } \mathrm{C} \\ \mathrm{TnI} & =\text { Troponin I } \\ \mathrm{TnT} & =\text { Troponin } \mathrm{T} \\ \mathrm{Xb} & =\text { Cross-bridge } \\ {[\mathrm{Xb}]} & =\text { Cross-bridge concentration }\end{array}$

\section{APPENDIX}

\section{Glossary}

A: actin

$\alpha$ : sarcomere overlap function

$\mathrm{A}_{\mathrm{f}}$ : force factor

B: proportionality factor; contains myosin isoform characteristic

$F_{b}$ : active force

$\mathrm{F}_{\text {ext }}$ : external force (load)
$\mathrm{F}_{\mathrm{p}}$ : passive force

$F_{p E x}: F_{p}$ contribution from collagen

$\mathrm{F}_{\mathrm{pIF}}: \mathrm{F}_{\mathrm{p}}$ contribution from intermediate filaments

$F_{p T i t i n}: F_{p}$ contribution from titin

$\mathrm{h}$ : $\mathrm{Xb}$ elongation

$h_{\mathrm{c}}$ : steady state $\mathrm{Xb}$ elongation

$\mathrm{k}_{12}$ : myosin binding rate parameter

$\mathrm{k}_{-12}$ : myosin detachment rate parameter

$\mathrm{k}_{3}$ : rate parameter for the strong $\mathrm{Xb}$ formation

$\mathrm{k}_{-3}$ : rate parameter for the transition from the strong to the weak $\mathrm{Xb}$

$\mathrm{k}_{3 \mathrm{c}}$ : factor for $\mathrm{k}_{3}, \mathrm{k}_{3}$ at resting level

$\mathrm{k}_{3 \mathrm{f}}$ : factor determining the positive cooperativity caused by neighboring activated RUs affecting the strong $\mathrm{Xb}$ formation

$\mathrm{k}_{3 \mathrm{xb}}$ : factor for negative cooperativity caused by strong Xbs affecting the strong $\mathrm{Xb}$ formation

$\mathrm{k}_{-4}$ : $\mathrm{P}_{\mathrm{i}}$ binding rate parameter

$\mathrm{k}_{4}: \mathrm{P}_{\mathrm{i}}$ release rate parameter

$\mathrm{k}_{5}$ : ADP release rate parameter

$\mathrm{k}_{\text {loadTM}}$ : factor expressing the load dependence for the Tm conformational change to the "on" conformation at low load shortening

$\mathrm{K}_{\mathrm{m}}$ : SR pump parameter

$\mathrm{k}_{\text {off }}: \mathrm{Ca}^{2+}$ detachment rate constant

$\mathrm{k}_{\mathrm{OFF}}$ : factor for the positive cooperativity caused by neighboring RUs in the activated conformation

$\mathrm{k}_{\text {offi }}$ : factor for $\mathrm{k}_{\text {offf }}, \mathrm{k}_{\text {offI }}$ at resting level

$\mathrm{k}_{\text {offl }}$ : rate for the TnI conformational change to the "off" conformation

$\mathrm{k}_{\text {offrelax }}$ : factor for $\mathrm{k}_{\text {offTnI }}$

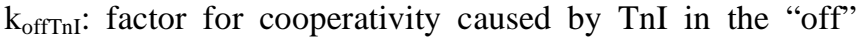
conformation pulling Tm to the "off" conformation

$\mathrm{k}_{\text {on }}: \mathrm{Ca}^{2+}$ binding rate constant

$\mathrm{k}_{\text {onI }}$ : rate for the TnI conformational change to the "on" conformation

$\mathrm{K}_{\mathrm{p}}$ : maximum value of $\mathrm{Q}_{\text {pump }}$

$\mathrm{k}_{\text {relaxSR }}$ : factor to enhance $\mathrm{Ca}^{2+}$ uptake during relaxation

$\mathrm{k}_{\text {relaxTnI: }}$ factor for enhancing the TnI conformational change to the "off" conformation

kTitin: scaling factor for the titin function

KTitin: titin function

$\mathrm{k}_{\text {tmoff: }}$ : rate parameter for the Tm conformational change to the "off" conformation

$\mathrm{k}_{\text {tmon }}$ : rate parameter for the Tm conformational change to the "on" conformation 
$\mathrm{k}_{\text {tmonc }}$ : factor for $\mathrm{k}_{\mathrm{tmon}}, \mathrm{k}_{\mathrm{tmon}}$ at resting level

$\mathrm{k}_{\mathrm{tmRU}}$ : factor determining the positive cooperativity caused by neighboring activated RUs affecting the Tm conformational change to the "on" conformation

$\mathrm{k}_{\mathrm{tmxb}}$ : factor for negative cooperativity caused by strong Xbs affecting the Tm conformational change to the "on" conformation

$\mathrm{k}_{\mathrm{xboff}}$ : factor for the positive cooperativity caused by strong Xbs

L: half sarcomere length

$\mathrm{L}_{0}$ : normalization factor

$\mathrm{L}_{1}$ : length change due to muscle elasticity in force step P1

$\mathrm{L}_{2}$ : sum of $\mathrm{L}_{1}$ plus $\mathrm{L}_{\mathrm{T}}$

$\mathrm{L}_{\mathrm{T}}$ : myosin working stroke, length change in P2

load: load fraction

M: myosin

$\mathrm{Q}_{\mathrm{ADPR}}$ : net rate of the ADP release step

$\mathrm{Q}_{\mathrm{CaB}}$ : net rate for $\mathrm{Ca}^{2+}$ binding to $\mathrm{TnC}$

$\mathrm{Q}_{\mathrm{Fgen}}$ : net rate of the force generating step

$\mathrm{Q}_{\mathrm{m}}$ : maximum $\mathrm{Q}_{\text {rel }}$ value

$\mathrm{Q}_{\mathrm{MB}}$ : net rate of the myosin binding step

$\mathrm{Q}_{\mathrm{PiR}}$ : net rate of the $\mathrm{P}_{\mathrm{i}}$ release step

$\mathrm{Q}_{\text {pump }}$ : SR Ca ${ }^{2+}$ uptake net rate

$\mathrm{Q}_{\text {pumprest }}: \mathrm{Q}_{\text {pump }}$ under resting conditions

$\mathrm{Q}_{\text {rel }}: \mathrm{Ca}^{2+}$ release from the $\mathrm{SR}$

$\mathrm{Q}_{\mathrm{TCaA}}$ : net rate for the $\mathrm{TnI}$ conformational change

$\mathrm{Q}_{\mathrm{TMA}}$ : net rate of the Tm conformational change

$\mathrm{RU}$ : regulatory unit

RUA: activated RUs

RUA_MADP: strong $\mathrm{Xb}, \mathrm{P}_{\mathrm{i}}$ released from the acto-myosin complex

RUA_MADPPi: strong Xb, M pocket for $\mathrm{P}_{\mathrm{i}}$ open

RUAMADPPi: $M$ with a hydrolyzed ATP bound to A of a activated RU (weak $\mathrm{Xb}$ ); $\mathrm{M}$ pocket for $\mathrm{P}_{\mathrm{i}}$ closed

RUNA: not activated RU

RUTCaoff: $\mathrm{Ca}^{2+}$ bound to TnC with TnI still bound to A

RUTCaon: TnI released from A

RUTMon: Tm in the activated conformation

RUtotal: sum of all RUs

t: time

$\mathrm{t}_{1}$ : time to $\mathrm{Qm}_{\mathrm{m}}$

$\mathrm{t}_{2}$ : force step phase 2 time interval

titinRF: factor expressing the effect from titin restoring force during relaxation
Tm: tropomyosin

TnC: troponin C

TnI: troponin I

V2: force step phase 2 shortening velocity

$\mathrm{X}$ : part of $\mathrm{L}$ which is not covered by $\mathrm{h}$

\section{REFERENCES}

[1] Norton JM. Toward consistent definitions for preload and afterload. Adv Physiol Educ 2001; 25: 53-61.

[2] Sonnenblick EH, Parmley WW, Urschel CW. The contractile state of the heart as expressed by force-velocity relations. Am J Cardiol 1969; 23: 488-503.

[3] Brutsaert DL, Sonnenblick EH. Force-velocity-length-time relations of the contractile elements in heart muscle of the cat. Circ Res 1969; 24: 137-49.

[4] de Tombe PP, ter Keurs HE. Sarcomere dynamics in cat cardiac trabeculae. Circ Res 1991; 68: 588-96.

[5] Piazzesi G, Lucii L, Lombardi V. The size and the speed of the working stroke of muscle myosin and its dependence on the force. $\mathrm{J}$ Physiol 2002; 545: 145-51.

[6] Edman KA, Mansson A, Caputo C. The biphasic force-velocity relationship in frog muscle fibres and its evaluation in terms of cross-bridge function. J Physiol 1997; 503: 141-56.

[7] Huxley AF. Muscle structure and theories of contraction. Prog Biophys Biophys Chem 1957; 7: 255-318.

[8] Duke TA. Molecular model of muscle contraction. Proc Natl Acad Sci U S A 1999; 96: 2770-5.

[9] Lan G, Sun SX. Dynamics of myosin-driven skeletal muscle contraction: I. Steady-state force generation. Biophys J 2005; 88: 4107-17.

[10] Chin L, Yue P, Feng JJ, Seow CY. Mathematical simulation of muscle cross-bridge cycle and force-velocity relationship. Biophys J 2006; 91: 3653-63.

[11] Negroni JA, Lascano EC. A cardiac muscle model relating sarcomere dynamics to calcium kinetics. J Mol Cell Cardiol 1996; 28 : 915-29.

[12] Negroni JA, Lascano EC. Simulation of steady state and transient cardiac muscle response experiments with a Huxley-based contraction model. J Mol Cell Cardiol 2008; 45: 300-12.

[13] Rice JJ, Wang F, Bers DM, de Tombe PP. Approximate model of cooperative activation and crossbridge cycling in cardiac muscle using ordinary differential equations. Biophys J 2008; 95: 2368-90.

[14] Schneider NS, Shimayoshi T, Amano A, Matsuda T. Mechanism of the Frank-Starling law--a simulation study with a novel cardiac muscle contraction model that includes titin and troponin I. J Mol Cell Cardiol 2006; 41: 522-36.

[15] Hanft LM, Korte FS, McDonald KS. Cardiac function and modulation of sarcomeric function by length. Cardiovasc Res 2008; 77: 627-36.

[16] Matsuoka S, Sarai N, Kuratomi S, Ono K, Noma A. Role of individual ionic current systems in ventricular cells hypothesized by a model study. Jpn J Physiol 2003; 53: 105-23.

[17] Gordon AM, Homsher E, Regnier M. Regulation of contraction in striated muscle. Physiol Rev 2000; 80: 853-924.

[18] Sasaki N, Mitsuiye T, Noma A, Powell T. Sarcomere length during contraction of isolated guinea-pig ventricular myocytes. Pflugers Arch 1999; 437: 804-11.

[19] Granzier HL, Irving TC. Passive tension in cardiac muscle: contribution of collagen, titin, microtubules, and intermediate filaments. Biophys J 1995; 68: 1027-44.

[20] Nair P, Wu Y, Helmes M, et al. Restoring force development by titin/connectin and assessment of Ig domain unfolding. J Muscle Res Cell Motil 2005; 26: 307-17.

[21] Helmes M, Lim CC, Liao R, et al. Titin determines the FrankStarling relation in early diastole. J Gen Physiol 2003; 121: 97-110. Reconditi M, Linari M, Lucii L, et al. Structure-function relation of the myosin motor in striated muscle. Ann N Y Acad Sci 2005; 1047: 232-47.

[23] Linari M, Brunello E, Reconditi M, et al. The structural basis of the increase in isometric force production with temperature in frog skeletal muscle. J Physiol 2005; 567: 459-69. 
[24] Decostre V, Bianco P, Lombardi V, Piazzesi G. Effect of temperature on the working stroke of muscle myosin. Proc Natl Acad Sci USA 2005; 102: 13927-32.

[25] Mathur AB, Collinsworth AM, Reichert WM, Kraus WE, Truskey GA. Endothelial, cardiac muscle and skeletal muscle exhibit different viscous and elastic properties as determined by atomic force microscopy. J Biomech 2001; 34: 1545-53.

[26] Colombini B, Nocella M, Benelli G, Cecchi G, Bagni MA. Effect of temperature on cross-bridge properties in intact frog muscle fibers. Am J Physiol 2008; 294: C1113-7.

[27] Piazzesi G, Reconditi M, Koubassova N, et al. Temperature dependence of the force-generating process in single fibres from frog skeletal muscle. J Physiol 2003; 549: 93-106.

[28] Sieck GC, Regnier M. Invited review: plasticity and energetic demands of contraction in skeletal and cardiac muscle. J Appl Physiol 2001; 90: 1158-64.

[29] Hinken AC, McDonald KS. Beta-myosin heavy chain myocytes are more resistant to changes in power output induced by ischemic conditions. Am J Physiol 2006; 290: H869-77.

[30] Donald TC, Reeves DN, Reeves RC, Walker AA, Hefner LL. Effect of damaged ends in papillary muscle preparations. Am J Physiol 1980; 238: H14-23.

[31] Brutsaert DL, Sonnenblick EH. Cardiac muscle mechanics in the evaluation of myocardial contractility and pump function: problems, concepts, and directions. Prog Cardiovasc Dis 1973; 16: 33761.

[32] Iribe G, Helmes M, Kohl P. Force-length relations in isolated intact cardiomyocytes subjected to dynamic changes in mechanical load. Am J Physiol 2007; 292: H1487-97.

[33] Sonnenblick EH, Skelton CL. Reconsideration of the ultrastructural basis of cardiac length-tension relations. Circ Res 1974; 35: 517 26.

[34] Fukuda N, Granzier HL, Ishiwata S, Kurihara S. Physiological functions of the giant elastic protein titin in Mammalian striated muscle. J Physiol Sci 2008; 58: 151-9.

[35] Murakami K, Yumoto F, Ohki SY, et al. Structural basis for $\mathrm{Ca}^{2+}-$ regulated muscle relaxation at interaction sites of troponin with actin and tropomyosin. J Mol Biol 2005; 352: 178-201.

[36] Sarai N, Matsuoka S, Noma A. simBio: a Java package for the development of detailed cell models. Prog Biophys Mol Biol 2006; 90: 360-77.

[37] Press WH, Flannery BP, Teukolsky SA, Vetterling WT. Numerical recipes in $\mathrm{C}$ : the art of scientific computing. 2nd ed. Cambridge University Press: Cambridge 1992.

[38] Brutsaert DL, Sys SU. Relaxation and diastole of the heart. Physiol Rev 1989; 69: 1228-315.

[39] Monasky MM, Varian KD, Davis JP, Janssen PM. Dissociation of force decline from calcium decline by preload in isolated rabbit myocardium. Pflugers Arch 2008; 456: 267-76.

[40] Wan X, Bryant SM, Hart G. A topographical study of mechanical and electrical properties of single myocytes isolated from normal guinea-pig ventricular muscle. J Anat 2003; 202: 525-36.

[41] Terui T, Sodnomtseren M, Matsuba D, et al. Troponin and titin coordinately regulate length-dependent activation in skinned porcine ventricular muscle. J Gen Physiol 2008; 131: 275-83.

[42] Balaban RS. Maintenance of the metabolic homeostasis of the heart: developing a systems analysis approach. Ann N Y Acad Sci 2006; 1080: 140-53.

[43] Robinson JM, Dong WJ, Xing J, Cheung HC. Switching of troponin $\mathrm{I}: \mathrm{Ca}(2+)$ and myosin-induced activation of heart muscle. $\mathbf{J}$ Mol Biol 2004; 340: 295-305.
[44] Nyitrai M, Geeves MA. Adenosine diphosphate and strain sensitivity in myosin motors. Philos Trans R Soc Lond B Biol Sci 2004; 359: 1867-77.

[45] Sleep J, Irving M, Burton K. The ATP hydrolysis and phosphate release steps control the time course of force development in rabbit skeletal muscle. J Physiol 2005; 563: 671-87.

[46] Guo B, Guilford WH. Mechanics of actomyosin bonds in different nucleotide states are tuned to muscle contraction. Proc Natl Acad Sci U S A 2006; 103: 9844-9.

[47] Capitanio M, Canepari M, Cacciafesta P, et al. Two independent mechanical events in the interaction cycle of skeletal muscle myosin with actin. Proc Natl Acad Sci U S A 2006; 103: 87-92.

[48] Hinken AC, McDonald KS. Inorganic phosphate speeds loaded shortening in rat skinned cardiac myocytes. Am J Physiol 2004; 287: C500-7.

[49] Piazzesi G, Reconditi M, Linari M, et al. Skeletal muscle performance determined by modulation of number of myosin motors rather than motor force or stroke size. Cell 2007; 131: 784-95.

[50] Burkholder TJ, Lieber RL. Sarcomere length operating range of vertebrate muscles during movement. J Exp Biol 2001; 204: 152936.

[51] Brutsaert DL, de Clerck NM, Goethals MA, Housmans PR. Relaxation of ventricular cardiac muscle. J Physiol 1978; 283: 469-80.

[52] Dobrunz LE, Berman MR. Effect of temperature on $\mathrm{Ca}^{(2+)}$ dependent and mechanical modulators of relaxation in mammalian myocardium. J Mol Cell Cardiol 1994; 26: 243-50.

[53] Neagoe C, Opitz CA, Makarenko I, Linke WA. Gigantic variety: expression patterns of titin isoforms in striated muscles and consequences for myofibrillar passive stiffness. J Muscle Res Cell Motil 2003; $24: 175-89$.

[54] McDonald KS, Moss RL. Strongly binding myosin crossbridges regulate loaded shortening and power output in cardiac myocytes. Circ Res 2000; 87: 768-73.

[55] Borlaug BA, Kass DA. Mechanisms of diastolic dysfunction in heart failure. Trends Cardiovasc Med 2006; 16: 273-9.

[56] Poggesi C, Tesi C, Stehle R. Sarcomeric determinants of striated muscle relaxation kinetics. Pflugers Arch 2005; 449: 505-17.

[57] Galinska-Rakoczy A, Engel P, Xu C, et al. Structural basis for the regulation of muscle contraction by troponin and tropomyosin. J Mol Biol 2008; 379: 929-35.

[58] Iorga B, Blaudeck N, Solzin J, et al. Lys184 deletion in troponin I impairs relaxation kinetics and induces hypercontractility in murine cardiac myofibrils. Cardiovasc Res 2008; 77: 676-86.

[59] Solaro RJ, Rosevear P, Kobayashi T. The unique functions of cardiac troponin I in the control of cardiac muscle contraction and relaxation. Biochem Biophys Res Commun 2008; 369: 82-7.

[60] Takimoto E, Soergel DG, Janssen PM, et al. Frequency- and afterload-dependent cardiac modulation in vivo by troponin I with constitutively active protein kinase A phosphorylation sites. Circ Res 2004; 94: 496-504.

[61] Stuyvers BD, McCulloch AD, Guo J, Duff HJ, ter Keurs HE. Effect of stimulation rate, sarcomere length and $\mathrm{Ca}(2+)$ on force generation by mouse cardiac muscle. J Physiol 2002; 544: 817-30.

[62] Robinson JM, Wang Y, Kerrick WG, Kawai R, Cheung HC. Activation of striated muscle: nearest-neighbor regulatory-unit and cross-bridge influence on myofilament kinetics. J Mol Biol 2002; 322: 1065-88.

[63] Sachse FB, Glänzel KG, Seemann G. Modeling of protein interactions involved in cardiac tension development. Int J Bifurcat Chaos 2003; 13: 3561-78

[64] Gordon AM, Regnier M, Homsher E. Skeletal and cardiac muscle contractile activation: tropomyosin "rocks and rolls". News Physiol Sci 2001; 16: 49-55.

This is an open access article licensed under the terms of the Creative Commons Attribution Non-Commercial License (http://creativecommons.org/licenses/by-nc/3.0/) which permits unrestricted, non-commercial use, distribution and reproduction in any medium, provided the work is properly cited. 